\title{
On existence and concentration of solutions to a class of quasilinear problems involving the 1-Laplace operator
}

\author{
Claudianor O. Alves ${ }^{1} \cdot \operatorname{Marcos}$ T. O. Pimenta ${ }^{2}$
}

Received: 16 February 2017 / Accepted: 14 September 2017 / Published online: 30 September 2017

C) Springer-Verlag GmbH Germany 2017

\begin{abstract}
In this work we use variational methods to prove results on existence and concentration of solutions to a problem in $\mathbb{R}^{N}$ involving the 1-Laplacian operator. A thorough analysis on the energy functional defined in the space of functions of bounded variation $B V\left(\mathbb{R}^{N}\right)$ is necessary, where the lack of compactness is overcome by using the Concentration of Compactness Principle due to Lions.
\end{abstract}

Mathematics Subject Classification $35 \mathrm{~J} 62 \cdot 35 \mathrm{~J} 20$

\section{Introduction and some abstract results}

Several recent studies have focused on the nonlinear Schrödinger equation

$$
i \epsilon \frac{\partial \Psi}{\partial t}=-\epsilon^{2} \Delta \Psi+(V(z)+E) \Psi-f(\Psi) \text { for all } z \in \mathbb{R}^{N},
$$

where $N \geq 2, \epsilon>0$ is a positive parameter and $V, f$ are continuous function verifying some conditions. This class of equation is one of the main objects of the quantum physics, because it appears in problems involving nonlinear optics, plasma physics and condensed matter physics.

\section{Communicated by P. Rabinowitz.}

\} \text { Marcos T. O. Pimenta } pimenta@fct.unesp.br

Claudianor O. Alves coalves@mat.ufcg.edu.br

1 Unidade Acadêmica de Matemática, Universidade Federal de Campina Grande, Campina Grande, PB 58429-900, Brazil

2 Departamento de Matemática e Computação, Faculdade de Ciências e Tecnologia, Universidade Estadual Paulista (Unesp), Presidente Prudente, SP 19060-900, Brazil 
The knowledge of the solutions for the elliptic equation

$$
\left\{\begin{array}{l}
-\epsilon^{2} \Delta u+V(z) u=f(u) \text { in } \mathbb{R}^{N}, \\
u \in H^{1}\left(\mathbb{R}^{N}\right),
\end{array}\right.
$$

has a great importance in the study of standing-wave solutions of $(N L S)$. The existence and concentration of positive solutions for general semilinear elliptic equations $(S)_{\epsilon}$ for the case $N \geq 3$ have been extensively studied, see for example, Floer and Weinstein [15], Oh [21], Rabinowitz [22], Wang [24], Cingolani and Lazzo [7], Ambrosetti et al. [1], Gui [16], del Pino and Felmer [8] and their references.

In the above mentioned papers, the existence, multiplicity and concentration of positive solutions have been obtained in connection with the geometry of the function $V$. In [22], by a mountain pass argument, Rabinowitz proves the existence of positive solutions of $(S)_{\epsilon}$ for $\epsilon>0$ small and

$$
\liminf _{|z| \rightarrow \infty} V(z)>\inf _{z \in \mathbb{R}^{N}} V(z)=V_{0}>0 .
$$

Later Wang [24] showed that these solutions concentrate at global minimum points of $V$ as $\epsilon$ tends to 0. In [8], del Pino and Felmer have found solutions which concentrate around local minimum of $V$ by introducing a penalization method. More precisely, they assume that there is an open and bounded set $\Lambda \subset \mathbb{R}^{N}$ such that

$$
0<V_{0} \leq \inf _{z \in \Lambda} V(z)<\min _{z \in \partial \Lambda} V(z) .
$$

Motivated by papers [22,24], let us consider the following class of quasilinear elliptic problems

$$
\left\{\begin{array}{l}
-\epsilon \Delta_{1} u+V(x) \frac{u}{|u|}=f(u) \text { in } \mathbb{R}^{N}, \\
u \in B V\left(\mathbb{R}^{N}\right),
\end{array}\right.
$$

where $\epsilon>0, N \geq 2$ and the operator $\Delta_{1}$ is the well known 1-Laplacian operator, whose formal definition is given by $\Delta_{1} u=\operatorname{div}\left(\frac{\nabla u}{|\nabla u|}\right)$. The nonlinearity $f$ is assumed to satisfy the following set of assumptions:

$\left(f_{1}\right) \quad f \in C^{1}(\mathbb{R})$;

$\left(f_{2}\right) \quad f(s)=o(1)$ as $s \rightarrow 0$;

$\left(f_{3}\right)$ There exist constants $c_{1}, c_{2}>0$ and $p \in\left[1,1^{*}\right)$ such that

$$
|f(s)| \leq c_{1}+c_{2}|s|^{p-1} \quad \forall s \in \mathbb{R},
$$

where $1^{*}=\frac{N}{N-1}$

$\left(f_{4}\right)$ There exists $\theta>1$ such that

$$
0<\theta F(s) \leq f(s) s, \quad \text { for } s \neq 0,
$$

where $F(s)=\int_{0}^{s} f(t) d t$;

$\left(f_{5}\right) f$ is increasing.

Hereafter, the potential is going to be considered satisfying some of the following conditions:

$\left(V_{1}\right) \quad V \in L^{\infty}\left(\mathbb{R}^{N}\right)$ and $0<V_{0}:=\inf _{\mathbb{R}^{N}} V$;

$\left(V_{2}\right) \quad V_{\infty}:=\liminf _{|x| \rightarrow+\infty} V(x)>V_{0}$;

$\left(V_{3}\right) \quad V \in C\left(\mathbb{R}^{N}\right), \lim _{|x| \rightarrow+\infty} V(x)=V_{\infty}$ and $V \leq V_{\infty}$ in $\mathbb{R}^{N}$. 
Hereafter, we will say that $V$ satisfies the Rabinowitz's condition when $\left(V_{1}\right)-\left(V_{2}\right)$ hold.

By studying problem (1.1) we are looking to get some results on existence and concentration of solutions, as the parameter $\epsilon \rightarrow 0^{+}$. The approach used as in the laplacian case is variational. However, the right space in which problem (1.1) takes place is the space of functions of bounded variation, $B V\left(\mathbb{R}^{N}\right)$. The energy function associated to (1.1) is $I_{\epsilon}: B V\left(\mathbb{R}^{N}\right) \rightarrow \mathbb{R}$, defined by

$$
I_{\epsilon}(u)=\epsilon \int_{\mathbb{R}^{N}}|D u|+\int_{\mathbb{R}^{N}} V(x)|u| d x-\int_{\mathbb{R}^{N}} F(u) d x,
$$

where $|D u|$ is the total variation of the vectorial Radon measure $D u$ (see Sect. 2).

Before we state our main results, we would like to mention the main difficulties in dealing with (1.1), which are organized in the list bellow:

- Problem (1.1) is just a formal version of the correct Euler-Lagrange equation associated to the functional $I_{\epsilon}$, since it is not well defined wherever $\nabla u$ or $u$ vanishes. For more details about this subject see Sect. 2.1.

- The functional $I_{\epsilon}$ is not $C^{1}\left(B V\left(\mathbb{R}^{N}\right)\right)$ and then some other sense of critical point have to be considered. Since $I_{\epsilon}$ is written like the difference between a convex locally Lipschitz functional and a smooth one, the theory of sub-differential of Clarke (see [5,9]) can be applied. Following this theory, it is possible to define a sense of critical point, PalaisSmale sequence, etc., that provide us with the tools to carry a variational approach to (1.1);

- The space $B V\left(\mathbb{R}^{N}\right)$, the domain of $I_{\epsilon}$, is not reflexive neither uniformly convex. This is the reason why is so difficult to prove that the functionals defined in this space satisfy compactness conditions like the Palais-Smale one;

- The solutions we will get lacks smoothness, then a lot of arguments explored in the literature cannot be used here, like convergence in the sense $C_{l o c}^{2}\left(\mathbb{R}^{N}\right), C_{l o c}^{1}\left(\mathbb{R}^{N}\right)$, etc.

- To overcome the above difficulties we have used in Sect. 3 the Concentration of Compactness Principle due to Lions, which is in our opinion an important novelty in the study of concentration of solution. Here, we must observe that our approach can also be used for concentration problem involving the laplacian operator.

- Here to get a solution we must prove that if $\left(v_{n}\right)$ is a Palais-Smale sequence associated with the energy functional we must have

$$
\int_{\mathbb{R}^{N}} f\left(v_{n}\right) v_{n} d x \rightarrow \int_{\mathbb{R}^{N}} f(v) v d x .
$$

For a lot of problems involving the Laplacian the above limit is not necessary to get a nontrivial solution, however for our problem this limit is crucial.

Our main results are the following theorems.

Theorem 1 Suppose that $f$ satisfies the conditions $\left(f_{1}\right)-\left(f_{5}\right)$ and that $V$ satisfies $\left(V_{1}\right)$ and $\left(V_{2}\right)$. Then there exist $\epsilon_{0}>0$ such that (1.1) has a nontrivial bounded variation solution $u_{\epsilon}$, for all $0<\epsilon<\epsilon_{0}$. Moreover, for each sequence $\epsilon_{n} \rightarrow 0$, up to a subsequence, the family $\left(u_{\epsilon_{n}}\right)_{n \in \mathbb{N}}$ concentrate around a point $x_{0} \in \mathbb{R}^{N}$ such that $V\left(x_{0}\right)=V_{0}$. More specifically, there exists $C>0$ such that for all $\delta>0$, there exist $\bar{R}>0$ and $n_{0} \in \mathbb{N}$ such that,

$$
\int_{B_{\epsilon_{n} \bar{R}}^{c}\left(x_{0}\right)} f\left(u_{n}\right) u_{n} d x<\epsilon_{n}^{N} \delta \text { and } \int_{B_{\epsilon_{n}}\left(x_{0}\right)} f\left(u_{n}\right) u_{n} d x \geq C \epsilon_{n}^{N},
$$

for all $n \geq n_{0}$. 
Our second result shows the existence of solution for all $\epsilon>0$ when $V$ is asymptotically constant and it has the following statement.

Theorem 2 Suppose that $f$ and $V$ satisfy the conditions $\left(f_{1}\right)-\left(f_{5}\right)$ and also $\left(V_{1}\right)$ and $\left(V_{3}\right)$, then there exist a nontrivial bounded variation solution $u_{\epsilon}$ of (1.1) for all $\epsilon>0$.

Here, we would like point out that a version of Theorem 2 for Laplacian operator was proved by Jianfu and Xiping [18].

Before concluding this section, we would like to mention some papers involving the $\Delta_{1}$ on bounded domains, where the reader can find more references about this subject. In [10], Degiovanni and Magrone study the version of Brézis-Nirenberg problem to the 1-Laplacian operator, corresponding to

$$
\left\{\begin{array}{l}
-\Delta_{1} u=\lambda \frac{u}{|u|}+|u|^{1^{*}-2} u \text { in } \Omega \\
u=0 \text { on } \partial \Omega
\end{array}\right.
$$

In [6], Chang uses this approach to study the spectrum of the 1-Laplacian operator, proving the existence of a sequence of eigenvalues. In [19], Kawohl and Schuricht also study the spectrum of the 1-Laplacian operator and reach the astonishing conclusion that an eigenfunction of this operator, in general satisfies infinity many Euler-Lagrange equations associated with it. In unbounded domains, in [13], Figueiredo and Pimenta dealt with an autonomous problem related to (1.1) by proving a version in the space $B V\left(\mathbb{R}^{N}\right)$ of a classical compactness result. In [14], Figueiredo and Pimenta study a non-perturbed version of (1.1) in the presence of vanishing potentials, by proving a version of the Mountain Pass Theorem without the PalaisSmale condition to functionals written like the difference between a locally Lipschitz and a smooth one.

The paper is organized as follows. In Sect. 2 we give a brief overview about the space $B V\left(\mathbb{R}^{N}\right)$, define the sense of solution we are going to deal with and also find the precise Euler-Lagrange equation associated to $I_{\epsilon}$. In Sect. 3 we prove Theorem 1, studying separately the arguments on existence and concentration of the solutions. In Sect. 4 we prove Theorem 2. Finally, in the last section we prove the existence of a ground-state solution to the autonomous problem.

\section{Preliminary results}

First of all let us note that the problem (1.1), through the change of variable $v(x)=u(\epsilon x)$, is equivalent to the problem

$$
\left\{\begin{array}{l}
-\Delta_{1} v+V(\epsilon x) \frac{v}{|v|}=f(v) \text { in } \mathbb{R}^{N}, \\
u \in B V\left(\mathbb{R}^{N}\right) .
\end{array}\right.
$$

Let us introduce the space of functions of bounded variation, $B V\left(\mathbb{R}^{N}\right)$. We say that $u \in B V\left(\mathbb{R}^{N}\right)$, or is a function of bounded variation, if $u \in L^{1}\left(\mathbb{R}^{N}\right)$, and its distributional derivative $D u$ is a vectorial Radon measure, i.e.,

$$
B V\left(\mathbb{R}^{N}\right)=\left\{u \in L^{1}\left(\mathbb{R}^{N}\right) ; D u \in \mathcal{M}\left(\mathbb{R}^{N}, \mathbb{R}^{N}\right)\right\} .
$$

It can be proved that $u \in B V\left(\mathbb{R}^{N}\right)$ is equivalent to $u \in L^{1}\left(\mathbb{R}^{N}\right)$ and

$$
\int_{\mathbb{R}^{N}}|D u|:=\sup \left\{\int_{\mathbb{R}^{N}} u \operatorname{div} \phi d x ; \phi \in C_{c}^{1}\left(\mathbb{R}^{N}, \mathbb{R}^{N}\right) \text {, s.t. }|\phi|_{\infty} \leq 1\right\}<+\infty .
$$


The space $B V\left(\mathbb{R}^{N}\right)$ is a Banach space when endowed with the norm

$$
\|u\|:=\int_{\mathbb{R}^{N}}|D u|+|u|_{1},
$$

which is continuously embedded into $L^{r}\left(\mathbb{R}^{N}\right)$ for all $r \in\left[1,1^{*}\right]$.

As one can see in [3], the space $B V\left(\mathbb{R}^{N}\right)$ has different convergence and density properties than the usual Sobolev spaces. For example, $C_{0}^{\infty}\left(\mathbb{R}^{N}\right)$ is not dense in $B V\left(\mathbb{R}^{N}\right)$ with respect to the strong convergence, since $\overline{C_{0}^{\infty}\left(\mathbb{R}^{N}\right)}$ w.r.t. the $B V\left(\mathbb{R}^{N}\right)$ norm is equal to $W^{1,1}\left(\mathbb{R}^{N}\right)$, a proper subspace of $B V\left(\mathbb{R}^{N}\right)$. This has motivated people to define a weaker sense of convergence in $B V\left(\mathbb{R}^{N}\right)$, called intermediate convergence. We say that $\left(u_{n}\right) \subset B V\left(\mathbb{R}^{N}\right)$ converge to $u \in B V\left(\mathbb{R}^{N}\right)$ in the sense of the intermediate convergence if

$$
u_{n} \rightarrow u, \quad \text { in } L^{1}\left(\mathbb{R}^{N}\right)
$$

and

$$
\int_{\mathbb{R}^{N}}\left|D u_{n}\right| \rightarrow \int_{\mathbb{R}^{N}}|D u|
$$

as $n \rightarrow \infty$. Fortunately, with respect to the intermediate convergente, $C_{0}^{\infty}\left(\mathbb{R}^{N}\right)$ is dense in $B V\left(\mathbb{R}^{N}\right)$.

For a vectorial Radon measure $\mu \in \mathcal{M}\left(\mathbb{R}^{N}, \mathbb{R}^{N}\right)$, we denote by $\mu=\mu^{a}+\mu^{s}$ the usual decomposition stated in the Radon Nikodyn Theorem, where $\mu^{a}$ and $\mu^{s}$ are, respectively, the absolute continuous and the singular parts with respect to the $N$-dimensional Lebesgue measure $\mathcal{L}^{N}$. We denote by $|\mu|$, the absolute value of $\mu$, the scalar Radon measure defined like in [3, p. 125]. By $\frac{\mu}{|\mu|}(x)$ we denote the usual Lebesgue derivative of $\mu$ with respect to $|\mu|$, given by

$$
\frac{\mu}{|\mu|}(x)=\lim _{r \rightarrow 0} \frac{\mu\left(B_{r}(x)\right)}{|\mu|\left(B_{r}(x)\right)} .
$$

It can be proved that $\mathcal{J}: B V\left(\mathbb{R}^{N}\right) \rightarrow \mathbb{R}$, given by

$$
\mathcal{J}(u)=\int_{\mathbb{R}^{N}}|D u|+\int_{\mathbb{R}^{N}}|u| d x,
$$

is a convex functional and Lipschitz continuous in its domain. It is also well know that $\mathcal{J}$ is lower semicontinuous with respect to the $L^{r}\left(\mathbb{R}^{N}\right)$ topology, for $r \in\left[1,1^{*}\right]$ (see [17] for example). Although non-smooth, the functional $\mathcal{J}$ admits some directional derivatives. More specifically, as is shown in [2], given $u \in B V\left(\mathbb{R}^{N}\right)$, for all $v \in B V\left(\mathbb{R}^{N}\right)$ such that $(D v)^{S}$ is absolutely continuous w.r.t. $(D u)^{s}$ and such that $v$ is equal to 0 a.e. in the set where $u$ vanishes, it follows that

$$
\mathcal{J}^{\prime}(u) v=\int_{\mathbb{R}^{N}} \frac{(D u)^{a}(D v)^{a}}{\left|(D u)^{a}\right|} d x+\int_{\mathbb{R}^{N}} \frac{D u}{|D u|}(x) \frac{D v}{|D v|}(x)|(D v)|^{s}+\int_{\mathbb{R}^{N}} \operatorname{sgn}(u) v d x,
$$

where $\operatorname{sgn}(u(x))=0$ if $u(x)=0$ and $\operatorname{sgn}(u(x))=u(x) /|u(x)|$ if $u(x) \neq 0$. In particular, note that, for all $u \in B V\left(\mathbb{R}^{N}\right)$,

$$
\mathcal{J}^{\prime}(u) u=\mathcal{J}(u)
$$


Let us define in the space $B V\left(\mathbb{R}^{N}\right)$ the following norms,

$$
\begin{aligned}
\|v\|_{\epsilon} & :=\int_{\mathbb{R}^{N}}|D v|+\int_{\mathbb{R}^{N}} V(\epsilon x)|v| d x, \\
\|v\|_{\infty} & :=\int_{\mathbb{R}^{N}}|D v|+\int_{\mathbb{R}^{N}} V_{\infty}|v| d x,
\end{aligned}
$$

and

$$
\|v\|_{0}:=\int_{\mathbb{R}^{N}}|D v|+\int_{\mathbb{R}^{N}} V_{0}|v| d x,
$$

which by $\left(V_{1}\right)$ and, $\left(V_{2}\right)$ or $\left(V_{3}\right)$, are equivalent to the usual norm in $B V\left(\mathbb{R}^{N}\right)$.

Let us define also the functionals $\Phi_{\epsilon}, \Phi_{\infty}, \Phi_{0}: B V\left(\mathbb{R}^{N}\right) \rightarrow \mathbb{R}$ by

$$
\begin{gathered}
\Phi_{\epsilon}(v)=\|v\|_{\epsilon}-\int_{\mathbb{R}^{N}} F(v) d x, \\
\Phi_{\infty}(v)=\|v\|_{\infty}-\int_{\mathbb{R}^{N}} F(v) d x
\end{gathered}
$$

and

$$
\Phi_{0}(v)=\|v\|_{0}-\int_{\mathbb{R}^{N}} F(v) d x .
$$

Denoting $\mathcal{F}(v)=\int_{\mathbb{R}^{N}} F(v) d x$ and $\mathcal{J}_{\epsilon}(v)=\|v\|_{\epsilon}$, note that $\mathcal{F} \in C^{1}\left(B V\left(\mathbb{R}^{N}\right)\right)$ and $\mathcal{J}_{\epsilon}$ defines a locally Lipschitz continuous functional. Then we say that $v_{\epsilon} \in B V\left(\mathbb{R}^{N}\right)$ is a solution of (2.1) if $0 \in \partial \Phi_{\epsilon}\left(v_{\epsilon}\right)$, where $\partial \Phi_{\epsilon}\left(v_{\epsilon}\right)$ denotes the generalized gradient of $\Phi_{\epsilon}$ in $v_{\epsilon}$, as defined in [5]. It follows that this is equivalent to $\mathcal{F}^{\prime}\left(v_{\epsilon}\right) \in \partial \mathcal{J}_{\epsilon}\left(v_{\epsilon}\right)$ and, since $\mathcal{J}_{\epsilon}$ is convex, this is written as

$$
\|w\|_{\epsilon}-\left\|v_{\epsilon}\right\|_{\epsilon} \geq \int_{\mathbb{R}^{N}} f\left(v_{\epsilon}\right)\left(w-v_{\epsilon}\right) d x, \quad \forall w \in B V\left(\mathbb{R}^{N}\right) .
$$

Hence all $v_{\epsilon} \in B V\left(\mathbb{R}^{N}\right)$ such that (2.5) holds is going to be called a bounded variation solution of (2.1). Analogously we define critical points of the functionals $\Phi_{\infty}$ and $\Phi_{0}$, since they have the same properties that $\Phi_{\epsilon}$.

\subsection{The Euler-Lagrange equation}

Since (2.1) contains expressions that doesn't make sense when $\nabla u=0$ or $u=0$, then it can be understood just as the formal version of the Euler-Lagrange equation associated to the functional $\Phi_{\epsilon}$. In this section we present the precise form of an Euler-Lagrange equation satisfied by all bounded variation critical points of $\Phi_{\epsilon}$. In order to do so we closely follow the arguments in [19], however we have introduced new ideas, because we are working in whole $\mathbb{R}^{N}$.

The first step is to consider the extension of the functionals $\mathcal{J}_{\epsilon}, \mathcal{F}$ and $\Phi_{\epsilon}$ to $X=$ $L^{1}\left(\mathbb{R}^{N}\right) \cap L^{\frac{N}{N-1}}\left(\mathbb{R}^{N}\right)$ endowed with the norm $\|w\|_{X}=|w|_{1}+|w|_{\frac{N}{N-1}}$, given respectively by $\overline{\mathcal{J}}_{\epsilon}, \overline{\mathcal{F}}, \bar{\Phi}_{\epsilon}: X \rightarrow \mathbb{R} \cup\{+\infty\}$, where

$$
\begin{aligned}
& \overline{\mathcal{J}}_{\epsilon}(v)= \begin{cases}\mathcal{J}_{\epsilon}(v), & \text { if } v \in B V\left(\mathbb{R}^{N}\right), \\
+\infty, & \text { if } v \in X \backslash B V\left(\mathbb{R}^{N}\right),\end{cases} \\
& \overline{\mathcal{F}}(u)=\int_{\mathbb{R}^{N}} F(u) d x
\end{aligned}
$$


and $\bar{\Phi}_{\epsilon}=\overline{\mathcal{J}}_{\epsilon}-\overline{\mathcal{F}}$. It is easy to see that $\overline{\mathcal{F}}$ belongs to $C^{1}(X, \mathbb{R})$ and that $\overline{\mathcal{J}}_{\epsilon}$ is a convex lower semicontinuous functional defined in $X$. Hence the subdifferential (in the sense of [23]) of $\overline{\mathcal{J}}_{\epsilon}$, denoted by $\partial \overline{\mathcal{J}}_{\epsilon}$, is well defined. The following is a crucial result in obtaining an Euler-Lagrange equation satisfied by the critical points of $\Phi_{\epsilon}$.

Lemma 3 If $v_{\epsilon} \in B V\left(\mathbb{R}^{N}\right)$ is such that $0 \in \partial \Phi_{\epsilon}\left(v_{\epsilon}\right)$, then $0 \in \partial \overline{\Phi_{\epsilon}}\left(v_{\epsilon}\right)$.

Proof Suppose that $0 \in \partial \Phi_{\epsilon}\left(v_{\epsilon}\right)$, i.e., that $v_{\epsilon}$ satisfies (2.5). We would like to prove that

$$
\overline{\mathcal{J}}_{\epsilon}(w)-\overline{\mathcal{J}}_{\epsilon}\left(v_{\epsilon}\right) \geq \overline{\mathcal{F}}^{\prime}\left(v_{\epsilon}\right)\left(w-v_{\epsilon}\right), \quad \forall w \in X .
$$

To see why, consider $w \in X$ and note that:

- if $w \in B V\left(\mathbb{R}^{N}\right) \cap X$, then

$$
\begin{aligned}
\overline{\mathcal{J}}_{\epsilon}(w)-\overline{\mathcal{J}}_{\epsilon}\left(v_{\epsilon}\right) & =\mathcal{J}_{\epsilon}(w)-\mathcal{J}_{\epsilon}\left(v_{\epsilon}\right) \\
& \geq \mathcal{F}^{\prime}\left(v_{\epsilon}\right)\left(w-v_{\epsilon}\right) \\
& =\int_{\mathbb{R}^{N}} f\left(v_{\epsilon}\right)\left(w-v_{\epsilon}\right) d x \\
& =\overline{\mathcal{F}}^{\prime}\left(v_{\epsilon}\right)\left(w-v_{\epsilon}\right) ;
\end{aligned}
$$

- if $w \in X \backslash B V\left(\mathbb{R}^{N}\right)$, since $\overline{\mathcal{J}}_{\epsilon}(w)=+\infty$ and $\overline{\mathcal{J}}_{\epsilon}\left(v_{\epsilon}\right)<+\infty$, it follows that

$$
\begin{aligned}
\overline{\mathcal{J}}_{\epsilon}(w)-\overline{\mathcal{J}}_{\epsilon}\left(v_{\epsilon}\right) & =+\infty \\
& \geq \overline{\mathcal{F}}^{\prime}\left(v_{\epsilon}\right)\left(w-v_{\epsilon}\right) .
\end{aligned}
$$

Therefore the result follows.

Let us assume that $v_{\epsilon} \in B V\left(\mathbb{R}^{N}\right)$ is a bounded variation solution of (2.1), i.e., that $v_{\epsilon}$ satisfies (2.5). Since $0 \in \partial \Phi_{\epsilon}\left(v_{\epsilon}\right)$, by the last result it follows that $0 \in \partial \bar{\Phi}_{\epsilon}\left(v_{\epsilon}\right)$. Since $\overline{\mathcal{J}}_{\epsilon}$ is convex and $\overline{\mathcal{F}}$ is smooth, it follows that $\overline{\mathcal{F}}^{\prime}\left(v_{\epsilon}\right) \in \partial \overline{\mathcal{J}}_{\epsilon}\left(v_{\epsilon}\right)$. In what follows, we set $\frac{\mathcal{J}_{\epsilon}^{1}}{,}, \overline{\mathcal{J}_{\epsilon}^{2}}: X \rightarrow \mathbb{R} \cup\{+\infty\}$ by

$$
\overline{\mathcal{J}_{\epsilon}^{1}}(v):= \begin{cases}\int_{\mathbb{R}^{N}}|D v|, & \text { if } v \in B V\left(\mathbb{R}^{N}\right), \\ +\infty, & \text { if } v \in X \backslash B V\left(\mathbb{R}^{N}\right),\end{cases}
$$

and

$$
\overline{\mathcal{J}_{\epsilon}^{2}}(v):=\int_{\mathbb{R}^{N}} V(\epsilon x)|v| d x .
$$

Note that $\overline{\mathcal{J}_{\epsilon}^{2}} \in C(X, \mathbb{R}), \overline{\mathcal{J}_{\epsilon}^{2}} \in C\left(B V\left(\mathbb{R}^{N}\right), \mathbb{R}\right)$ and

$$
\overline{\mathcal{J}_{\epsilon}}(v)=\overline{\mathcal{J}_{\epsilon}^{1}}(v)+\overline{\mathcal{J}_{\epsilon}^{2}}(v), \quad \forall v \in X .
$$

Since $\overline{\mathcal{J}_{\epsilon}^{2}}$ and $\overline{\mathcal{J}_{\epsilon}^{2}}$ are convex, and $\overline{\mathcal{J}_{\epsilon}^{2}}$ is finite and continuous in every point of $B V\left(\mathbb{R}^{N}\right)$, it follows from [3, Theorem 9.5.4] that

$$
\overline{\mathcal{F}}^{\prime}\left(v_{\epsilon}\right) \in \partial \overline{\mathcal{J}}_{\epsilon}\left(v_{\epsilon}\right)=\partial \overline{\mathcal{J}_{\epsilon}^{1}}\left(v_{\epsilon}\right)+\partial \overline{\mathcal{J}_{\epsilon}^{2}}\left(v_{\epsilon}\right)
$$

By using the same arguments explored in [4, Theorem 8.15], it follows that $X^{\prime} \subset L_{\infty, N}\left(\mathbb{R}^{N}\right)$ where

$$
L_{\infty, N}\left(\mathbb{R}^{N}\right)=\left\{g: \mathbb{R}^{N} \rightarrow \mathbb{R} \text { measurable }:\|g\|_{\infty, N}<\infty\right\}
$$


where

$$
\|g\|_{\infty, N}=\sup _{|\phi|_{1}+|\phi|_{\frac{N}{N-1}} \leq 1}\left|\int_{\mathbb{R}^{N}} g \phi d x\right| .
$$

It is possible to prove that \|\|$_{\infty, N}$ is a norm in $L_{\infty, N}\left(\mathbb{R}^{N}\right)$. Moreover, the inclusion $L_{\infty, N}\left(\mathbb{R}^{N}\right) \hookrightarrow L^{N}\left(B_{R}(0)\right)$ is continuous for all $R>0$.

From the above commentaries, there are $z_{1}^{*}, z_{2}^{*} \in L_{\infty, N}\left(\mathbb{R}^{N}\right)$ such that $z_{1}^{*} \in \partial \overline{\mathcal{J}_{\epsilon}^{1}}\left(v_{\epsilon}\right)$, $z_{2}^{*} \in \partial \overline{\mathcal{J}}_{\epsilon}^{2}\left(v_{\epsilon}\right)$ and

$$
\overline{\mathcal{F}}^{\prime}\left(v_{\epsilon}\right)=z_{1}^{*}+z_{2}^{*} \text { in } L_{\infty, N}\left(\mathbb{R}^{N}\right) .
$$

Following the same arguments in [19, Proposition 4.23, p. 529], we have that there exists $z \in L^{\infty}\left(\mathbb{R}^{N}, \mathbb{R}^{N}\right)$ such that $|z|_{\infty} \leq 1$,

$$
-\operatorname{div} z=z_{1}^{*} \text { in } L_{\infty, N}\left(\mathbb{R}^{N}\right)
$$

and

$$
-\int_{\mathbb{R}^{N}} v_{\epsilon} \operatorname{div} z d x=\int_{\mathbb{R}^{N}}\left|D v_{\epsilon}\right|,
$$

where the divergence in (2.6) has to be understood in the distributional sense. Moreover, the same result implies that $z_{2}^{*}$ is such that

$$
z_{2}^{*}\left|v_{\epsilon}\right|=V(\epsilon x) v_{\epsilon}, \quad \text { a.e. in } \mathbb{R}^{N} .
$$

Therefore, it follows from (2.6), (2.7) and (2.8) that $v_{\epsilon}$ satisfies

$$
\left\{\begin{array}{l}
\exists z \in L^{\infty}\left(\mathbb{R}^{N}, \mathbb{R}^{N}\right),\|z\|_{\infty} \leq 1, \operatorname{div} z \in L_{\infty, N}\left(\mathbb{R}^{N}\right),-\int_{\mathbb{R}^{N}} v_{\epsilon} \operatorname{div} z d x=\int_{\mathbb{R}^{N}}\left|D v_{\epsilon}\right|, \\
\exists z_{2}^{*} \in L_{\infty, N}\left(\mathbb{R}^{N}\right), z_{2}^{*}\left|v_{\epsilon}\right|=V(\epsilon x) v_{\epsilon} \quad \text { a.e. in } \mathbb{R}^{N}, \\
-\operatorname{div} z+z_{2}^{*}=f\left(v_{\epsilon}\right), \quad \text { a.e. in } \mathbb{R}^{N} .
\end{array}\right.
$$

Hence, (2.9) is the precise version of (1.1).

\section{Existence and concentration of solution with the Rabinowitz's condition}

Let us first observe that by standard calculations, it is possible to prove that $\Phi_{\epsilon}, \Phi_{\infty}$ and $\Phi_{0}$ satisfy the geometrical conditions of the Mountain Pass Theorem. Then the following minimax levels are well defined

$$
\begin{aligned}
c_{\epsilon} & =\inf _{\gamma \in \Gamma_{\epsilon}} \sup _{t \in[0,1]} \Phi_{\epsilon}(\gamma(t)), \\
c_{\infty} & =\inf _{\gamma \in \Gamma_{\infty}} \sup _{t \in[0,1]} \Phi_{\infty}(\gamma(t))
\end{aligned}
$$

and

$$
c_{0}=\inf _{\gamma \in \Gamma_{0}} \sup _{t \in[0,1]} \Phi_{0}(\gamma(t)),
$$

where $\Gamma_{\epsilon}=\left\{\gamma \in C\left([0,1], B V\left(\mathbb{R}^{N}\right) ; \gamma(0)=0\right.\right.$ and $\left.\Phi_{\epsilon}(\gamma(1))<0\right\}$ and $\Gamma_{\infty}, \Gamma_{0}$ are defined in an analogous way. Moreover, by study made in Sect. 5 , it follows that there exists a critical point of $\Phi_{\infty}, w_{\infty} \in B V\left(\mathbb{R}^{N}\right)$, such that $\Phi_{\infty}\left(w_{\infty}\right)=c_{\infty}$. By the same reason, there exists a critical point of $\Phi_{0}, w_{0} \in B V\left(\mathbb{R}^{N}\right)$, such that $\Phi_{0}\left(w_{0}\right)=c_{0}$. 
Let us define the Nehari manifolds associated to $\Phi_{\epsilon}, \Phi_{\infty}$ and $\Phi_{0}$, which are well defined by (2.4), respectively by

$$
\begin{aligned}
\mathcal{N}_{\epsilon} & =\left\{v \in B V\left(\mathbb{R}^{N}\right) \backslash\{0\} ; \Phi_{\epsilon}^{\prime}(v) v=0\right\}, \\
\mathcal{N}_{\infty} & =\left\{v \in B V\left(\mathbb{R}^{N}\right) \backslash\{0\} ; \Phi_{\infty}^{\prime}(v) v=0\right\}
\end{aligned}
$$

and

$$
\mathcal{N}_{0}=\left\{v \in B V\left(\mathbb{R}^{N}\right) \backslash\{0\} ; \Phi_{0}^{\prime}(v) v=0\right\}
$$

By the discussion in [12], it follows that $c_{\epsilon}=\inf _{\mathcal{N}_{\epsilon}} \Phi_{\epsilon}, c_{\infty}=\inf _{\mathcal{N}_{\infty}} \Phi_{\infty}$ and $c_{0}=$ $\inf _{\mathcal{N}_{0}} \Phi_{0}$.

\subsection{Existence results}

First of all we study the behavior of the minimax levels $c_{\epsilon}$, when $\epsilon \rightarrow 0^{+}$. For the sake of simplicity, let us suppose without lack of generality that $V(0)=V_{0}$.

Lemma $4 \lim _{\epsilon \rightarrow 0^{+}} c_{\epsilon}=c_{0}$.

Proof Let $\epsilon_{n} \rightarrow 0$ as $n \rightarrow+\infty$. Let $\psi \in C_{0}^{\infty}\left(\mathbb{R}^{N}\right)$ be such that $0 \leq \psi \leq 1, \psi \equiv 0$ in $\mathbb{R}^{N} / B_{2}(0), \psi \equiv 1$ in $B_{1}(0)$ and $|\nabla \psi| \leq C$ in $\mathbb{R}^{N}$. Let us define

$$
w_{\epsilon_{n}}(x)=\psi\left(\epsilon_{n} x\right) w_{0}(x),
$$

where $w_{0}$ is a ground state critical point of $\Phi_{0}$. Note that $w_{\epsilon_{n}} \rightarrow w_{0}$ in $B V\left(\mathbb{R}^{N}\right)$ and $\Phi_{0}\left(w_{\epsilon_{n}}\right) \rightarrow \Phi_{0}(w)$ as $n \rightarrow+\infty$. Let $t_{\epsilon_{n}}>0$ be such that $t_{\epsilon_{n}} w_{\epsilon_{n}} \in \mathcal{N}_{\epsilon_{n}}$ and let us suppose just for a while that $t_{\epsilon_{n}} \rightarrow 1$ as $n \rightarrow+\infty$. Then

$$
\begin{aligned}
c_{\epsilon_{n}} & \leq \Phi_{\epsilon_{n}}\left(t_{\epsilon_{n}} w_{\epsilon_{n}}\right) \\
& =\Phi_{0}\left(t_{\epsilon_{n}} w_{\epsilon_{n}}\right)+\int_{\mathbb{R}^{N}}\left(V\left(\epsilon_{n} x\right)-V_{0}\right) t_{\epsilon_{n}}\left|w_{\epsilon_{n}}\right| d x .
\end{aligned}
$$

Using the Lebesgue Dominated Theorem, it follows that

$$
\limsup _{n \rightarrow+\infty} c_{\epsilon_{n}} \leq \Phi_{0}\left(w_{0}\right)=c_{0}
$$
Then

On the other hand, since $\Phi_{0}(v) \leq \Phi_{\epsilon_{n}}(v)$ for all $v \in B V\left(\mathbb{R}^{N}\right)$, it follows that $c_{0} \leq c_{\epsilon_{n}}$.

$$
\lim _{n \rightarrow+\infty} c_{\epsilon_{n}}=c_{0}
$$

What is left to do is to prove that in fact $t_{\epsilon_{n}} \rightarrow 1$, as $n \rightarrow+\infty$. Since $\Phi_{\epsilon_{n}}^{\prime}\left(t_{\epsilon_{n}} w_{\epsilon_{n}}\right) w_{\epsilon_{n}}=0$, it follows that

$$
\int_{\mathbb{R}^{N}}\left|D w_{\epsilon_{n}}\right|+\int_{\mathbb{R}^{N}} V\left(\epsilon_{n} x\right)\left|w_{\epsilon_{n}}\right| d x=\int_{\mathbb{R}^{N}} f\left(t_{\epsilon_{n}} w_{\epsilon_{n}}\right) w_{\epsilon_{n}} d x .
$$

We claim that $\left(t_{\epsilon_{n}}\right)_{\epsilon_{n}>0}$ is bounded. In fact, on the contary, up to a subsequence, $t_{\epsilon_{n}} \rightarrow+\infty$. Let $\Sigma \subset \mathbb{R}^{N}$ be such that $|\Sigma|>0$ and $w_{0}(x) \neq 0$ for all $x \in \Sigma$. Hence it holds for all $n \in \mathbb{N}$ that 


$$
\begin{aligned}
\left\|w_{\epsilon_{n}}\right\|_{\epsilon_{n}} & =\int_{\mathbb{R}^{N}} \frac{f\left(t_{\epsilon_{n}} w_{\epsilon_{n}}\right) t_{\epsilon_{n}} w_{\epsilon_{n}}}{t_{\epsilon_{n}}} d x \\
& \geq \int_{\Sigma} \frac{\theta F\left(t_{\epsilon_{n}} w_{\epsilon_{n}}\right)}{t_{\epsilon_{n}}} d x .
\end{aligned}
$$

Then by $\left(f_{4}\right)$ and Fatou's Lemma it follows that

$$
\left\|w_{\epsilon_{n}}\right\|_{\epsilon_{n}} \rightarrow+\infty, \quad \text { as } n \rightarrow \infty,
$$

which contradicts the fact that $w_{\epsilon_{n}} \rightarrow w_{0}$ in $B V\left(\mathbb{R}^{N}\right)$ as $n \rightarrow \infty$.

Now we have to verify that $t_{\epsilon_{n}} \nrightarrow 0$ as $n \rightarrow+\infty$. In fact, on the contrary, from $\left(f_{2}\right)$ and the fact that $t_{\epsilon_{n}} w_{\epsilon_{n}} \in \mathcal{N}_{\epsilon_{n}}$, we would have that

$$
\left\|w_{\epsilon_{n}}\right\|_{\epsilon_{n}}=\int_{\mathbb{R}^{N}} f\left(t_{\epsilon_{n}} w_{\epsilon_{n}}\right) w_{\epsilon_{n}} d x=o_{n}(1),
$$

a clear contradiction. Then there exist $\alpha, \beta>0$ such that

$$
\alpha \leq t_{\epsilon_{n}} \leq \beta \text { for all } n \in \mathbb{N}
$$

and then, up to a subsequence, $t_{n} \rightarrow \bar{t}>0$, as $n \rightarrow+\infty$. Since

$$
\int_{\mathbb{R}^{N}}\left|D w_{\epsilon_{n}}\right|+\int_{\mathbb{R}^{N}} V\left(\epsilon_{n} x\right)\left|w_{\epsilon_{n}}\right| d x \rightarrow \int_{\mathbb{R}^{N}}\left|D w_{0}\right|+\int_{\mathbb{R}^{N}} V(0)\left|w_{0}\right| d x,
$$

from the definition of $w_{0}$, it follows by $\left(f_{5}\right)$ that $\bar{t}=1$.

Since by $\left(V_{2}\right), V_{0}<V_{\infty}$, it follows from the monotonicity of the energy functional w.r.t. the potentials that

$$
c_{0}<c_{\infty}
$$

As a consequence of Lemma 4 and (3.1), it holds the following result.

Corollary 5 There exists $\epsilon_{0}>0$ such that $c_{\epsilon}<c_{\infty}$ for all $\epsilon \in\left(0, \epsilon_{0}\right)$.

By [13, Theorem 4], for each $\epsilon>0$, there exists a Palais-Smale sequence $\left(v_{n}\right) \subset B V\left(\mathbb{R}^{N}\right)$ to $\Phi_{\epsilon}$ in the level $c_{\epsilon}$, i.e.

$$
\lim _{n \rightarrow \infty} \Phi_{\epsilon}\left(v_{n}\right)=c_{\epsilon}
$$

and

$$
\|w\|_{\epsilon}-\left\|v_{n}\right\|_{\epsilon} \geq \int_{\mathbb{R}^{N}} f\left(v_{n}\right)\left(w-v_{n}\right) d x-\tau_{n}\left\|w-v_{n}\right\|_{\epsilon}, \quad \forall w \in B V\left(\mathbb{R}^{N}\right),
$$

where $\tau_{n} \rightarrow 0$, as $n \rightarrow \infty$.

Lemma 6 The sequence $\left(v_{n}\right)$ is bounded in $B V\left(\mathbb{R}^{N}\right)$.

Proof Considering $w=2 v_{n}$ in (3.3), we get

$$
\left\|v_{n}\right\|_{\epsilon} \geq \int_{\mathbb{R}^{N}} f\left(v_{n}\right) v_{n} d x-\tau_{n}\left\|v_{n}\right\|_{\epsilon},
$$

which implies that

$$
\left(1+\tau_{n}\right)\left\|v_{n}\right\|_{\epsilon} \geq \int_{\mathbb{R}^{N}} f\left(v_{n}\right) v_{n} d x .
$$


Then, by $\left(f_{4}\right)$ and (3.4),

$$
\begin{aligned}
c_{\epsilon}+o_{n}(1) & \geq \Phi_{\epsilon}\left(v_{n}\right) \\
& =\left\|v_{n}\right\|_{\epsilon}+\int_{\mathbb{R}^{N}}\left(\frac{1}{\theta} f\left(v_{n}\right) v_{n}-F\left(v_{n}\right)\right) d x-\int_{\mathbb{R}^{N}} \frac{1}{\theta} f\left(v_{n}\right) v_{n} d x \\
& \geq\left\|v_{n}\right\|_{\epsilon}\left(1-\frac{1}{\theta}-\frac{\tau_{n}}{\theta}\right) \\
& \geq C\left\|v_{n}\right\|_{\epsilon},
\end{aligned}
$$

for some $C>0$ which does not depend on $n \in \mathbb{N}$. Then the result follows.

By the last result and the compactness of the embeddings of $B V\left(\mathbb{R}^{N}\right)$ in $L_{l o c}^{q}\left(\mathbb{R}^{N}\right)$ for $1 \leq q<1^{*}$, it follows that there exists $v_{\epsilon} \in B V_{l o c}\left(\mathbb{R}^{N}\right)$ such that

$$
v_{n} \rightarrow v_{\epsilon} \text { in } L_{l o c}^{q}\left(\mathbb{R}^{N}\right) \quad \text { for } 1 \leq q<1^{*}
$$

and

$$
v_{n} \rightarrow v_{\epsilon} \text { a.e. in } \mathbb{R}^{N}
$$

as $n \rightarrow+\infty$. Note that $v_{\epsilon} \in B V\left(\mathbb{R}^{N}\right)$. In fact, if $R>0$, by the semicontinuity of the norm in $B V\left(B_{R}(0)\right)$ w.r.t. the $L^{1}\left(B_{R}(0)\right)$ topology it follows that

$$
\left\|v_{\epsilon}\right\|_{B V\left(B_{R}(0)\right)} \leq \liminf _{n \rightarrow+\infty}\left\|v_{n}\right\|_{B V\left(B_{R}(0)\right)} \leq \liminf _{n \rightarrow+\infty}\left\|v_{n}\right\|_{B V\left(\mathbb{R}^{N}\right)} \leq C,
$$

where $C$ does not depend on $n$ or $R$. Since the last inequality holds for every $R>0$, then $v_{\epsilon} \in B V\left(\mathbb{R}^{N}\right)$.

The following is a crucial result in our argument. In its proof we use the well known Concentration of Compactness Principle due to Lions [20].

Proposition 7 If $\epsilon<\epsilon_{0}$, $\epsilon_{0}$ like in Corollary 5, then

$$
v_{n} \rightarrow v_{\epsilon} \text { in } L^{q}\left(\mathbb{R}^{N}\right) \text { for all } 1 \leq q<1^{*} .
$$

Proof Let us apply the Concentration of Compactness Principle of Lions to the following bounded sequence in $L^{1}\left(\mathbb{R}^{N}\right)$,

$$
\rho_{n}(x):=\frac{\left|v_{n}(x)\right|}{\left|v_{n}\right|_{1}}
$$

For future reference, note that

$$
\left|v_{n}\right|_{1} \not \rightarrow 0, \text { as } n \rightarrow+\infty .
$$

In fact, otherwise, by interpolation inequality $\left(v_{n}\right)$ would converge to 0 in $L^{q}\left(\mathbb{R}^{N}\right)$ for all $1 \leq q<1^{*}$. By taking $w=v_{n}+t v_{n}$ in (3.3) and doing $t \rightarrow 0$, it is easy to see that

$$
\left\|v_{n}\right\|_{\epsilon}=\int_{\mathbb{R}^{N}} f\left(v_{n}\right) v_{n} d x+o_{n}(1) .
$$

Then, by the last equality, $\left(f_{2}\right),\left(f_{3}\right)$ and the fact that $v_{n} \rightarrow 0$ in $L^{q}\left(\mathbb{R}^{N}\right)$ for all $1 \leq q<1^{*}$, the Lebesgue Convergence Theorem imply that $v_{n} \rightarrow 0$ in $B V\left(\mathbb{R}^{N}\right)$, implying that $c_{\epsilon}=0$, which contradicts the fact that $c_{\epsilon}>0$.

Since $\left(\rho_{n}\right)$ is a bounded sequence in $L^{1}\left(\mathbb{R}^{N}\right)$, the Concentration of Compactness Principle implies that one and only one of the following statements holds: 
(Vanishing) $\quad \lim _{n \rightarrow+\infty} \sup _{y \in \mathbb{R}^{N}} \int_{B_{R}(y)} \rho_{n} d x=0, \forall R>0 ;$

(Compactness) There exist $\left(y_{n}\right) \subset \mathbb{R}^{N}$ such that for all $\eta>0$, there exists $R>0$ such that

$$
\int_{B_{R}\left(y_{n}\right)} \rho_{n} d x \geq 1-\eta, \quad \forall n \in \mathbb{N} ;
$$

(Dichotomy) There exist $\left(y_{n}\right) \subset \mathbb{R}^{N}, \alpha \in(0,1), R_{1}>0, R_{n} \rightarrow+\infty$ such that the functions $\rho_{n, 1}(x):=\chi_{B_{R_{1}}\left(y_{n}\right)}(x) \rho_{n}(x)$ and $\rho_{n, 2}(x):=\chi_{B_{R_{n}}^{c}\left(y_{n}\right)}(x) \rho_{n}(x)$ satisfy

$$
\int_{\mathbb{R}^{N}} \rho_{1, n} d x \rightarrow \alpha \text { and } \int_{\mathbb{R}^{N}} \rho_{2, n} d x \rightarrow 1-\alpha .
$$

Our objective is to show that $\left(\rho_{n}\right)$ verifies the Compactness condition and in order to do so we act by excluding all the others possibilities.

Note that Vanishing does not occur. In fact, otherwise, by [13, Theorem 1.1], it would hold that $\rho_{n} \rightarrow 0$ in $L^{q}\left(\mathbb{R}^{N}\right)$, for all $1 \leq q<1^{*}$. Taking (3.8) into account, this would imply that $v_{n} \rightarrow 0$ in $L^{q}\left(\mathbb{R}^{N}\right)$, for all $1 \leq q<1^{*}$ and then, this would led us to $c_{\epsilon}=0$, a clear contradiction.

Let us show now that Dichotomy also does not hold. Firstly note that (3.3) implies that

$$
\Phi_{\epsilon}^{\prime}\left(v_{n}\right) v_{n}=o_{n}(1), \quad \text { as } n \rightarrow+\infty .
$$

As far as the sequence $\left(y_{n}\right)$ is concerned, let us consider the two possible situations.

- $\left(y_{n}\right)$ is bounded:

In this case the function $v_{\epsilon}$ is nontrivial, since

$$
\int_{B_{R}\left(y_{n}\right)} \frac{\left|v_{n}\right|}{\left|v_{n}\right|_{1}} d x \rightarrow \alpha,
$$

implies that

$$
\int_{B_{R}\left(y_{n}\right)}\left|v_{n}\right| d x \geq \delta, \quad \text { for all } n \text { sufficiently large. }
$$

Then, by taking $R_{0}>0$ such that $B_{R}\left(y_{n}\right) \subset B_{R_{0}}(0)$ for all $n \in \mathbb{N}$, it follows that

$$
\int_{B_{R_{0}}(0)}\left|v_{n}\right| d x \geq \delta, \quad \text { for all } n \text { sufficiently large, }
$$

implying by (3.5) that

$$
\int_{B_{R_{0}}(0)}\left|v_{\epsilon}\right| d x \geq \delta
$$

Now let us show the following claim.

Claim $1 \Phi_{\epsilon}^{\prime}\left(v_{\epsilon}\right) v_{\epsilon} \leq 0$.

Note that, if $\varphi \in C_{0}^{\infty}\left(\mathbb{R}^{N}\right), 0 \leq \varphi \leq 1, \varphi \equiv 1$ in $B_{R}(0)$ and $\varphi \equiv 0$ in $B_{2 R}(0)^{c}$, for $\varphi_{R}:=\varphi(\cdot / R)$, it follows that for all $v \in B V\left(\mathbb{R}^{N}\right)$,

$$
\left(D\left(\varphi_{R} v\right)\right)^{s} \text { is absolutely continuous w.r.t. }(D v)^{s} .
$$

In fact, note that

$$
D\left(\varphi_{R} v\right)=\nabla \varphi_{R} v+\varphi_{R} D v=\nabla \varphi_{R} v+\varphi_{R} D v^{a}+\varphi_{R} D v^{s}, \quad \text { in } \mathcal{D}^{\prime}\left(\mathbb{R}^{N}\right) .
$$


Then it follows that

$$
\left(D\left(\varphi_{R} v\right)\right)^{s}=\left(\varphi_{R}(D v)^{s}\right)^{s}=\varphi_{R}(D v)^{s} .
$$

Taking (3.14) into account, the fact that $\varphi_{R} v_{n}$ is equal to 0 a.e. in the set where $v_{n}$ vanishes and also the fact that $\frac{\varphi_{R} \mu}{\left|\varphi_{R} \mu\right|}=\frac{\mu}{|\mu|}$ a.e. in $B_{R}(0)$, it is well defined $\Phi_{\epsilon}^{\prime}\left(v_{n}\right)\left(\varphi_{R} v_{n}\right)$ and, by (2.3), it follows that

$$
\begin{aligned}
\Phi_{\epsilon}^{\prime}\left(v_{n}\right)\left(\varphi_{R} v_{n}\right)= & \int_{\mathbb{R}^{N}} \frac{\left(\left(D v_{n}\right)^{a}\right)^{2} \varphi_{R}+v_{n}\left(D v_{n}\right)^{a} \cdot \nabla \varphi_{R}}{\left|\left(D v_{n}\right)^{a}\right|} d x \\
& +\int_{\mathbb{R}^{N}} \frac{D v_{n}}{\left|D v_{n}\right|} \frac{\varphi_{R}\left(D v_{n}\right)^{s}}{\left|\varphi_{R}\left(D v_{n}\right)^{s}\right|}\left|\varphi_{R}\left(D v_{n}\right)^{s}\right| \\
& +\int_{\mathbb{R}^{N}} V(\epsilon x) \operatorname{sgn}\left(v_{n}\right)\left(\varphi_{R} v_{n}\right) d x-\int_{\mathbb{R}^{N}} f\left(v_{n}\right) \varphi_{R} v_{n} d x \\
= & \int_{\mathbb{R}^{N}} \varphi_{R}\left|\left(D v_{n}\right)^{a}\right| d x+\int_{\mathbb{R}^{N}} \frac{v_{n}\left(D v_{n}\right)^{a} \cdot \nabla \varphi_{R}}{\left|\left(D v_{n}\right)^{a}\right|} d x \\
& +\int_{\mathbb{R}^{N}} \frac{\left(D v_{n}\right)^{s}}{\left|\left(D v_{n}\right)^{s}\right|} \frac{\varphi_{R}\left(D v_{n}\right)^{s}}{\left|\varphi_{R}\left(D v_{n}\right)^{s}\right|}\left|\varphi_{R}\left(D v_{n}\right)^{s}\right|+\int_{\mathbb{R}^{N}} V(\epsilon x)\left|v_{n}\right| \varphi_{R} d x \\
& -\int_{\mathbb{R}^{N}} f\left(v_{n}\right) \varphi_{R} v_{n} d x .
\end{aligned}
$$

The last equality together with the lower semicontinuity of the norm in $B V\left(B_{R}(0)\right)$ w.r.t. the $L^{1}\left(B_{R}(0)\right)$ convergence and the fact that $\Phi_{\epsilon}^{\prime}\left(v_{n}\right)\left(\varphi_{R} v_{n}\right)=o_{n}(1)$ (since $\left(\varphi_{R} v_{n}\right)$ is bounded in $B V\left(\mathbb{R}^{N}\right)$ ), imply that

$$
\int_{B_{R}(0)}\left|D v_{\epsilon}\right|+\liminf _{n \rightarrow \infty} \int_{\mathbb{R}^{N}} \frac{v_{n}\left(D v_{n}\right)^{a} \cdot \nabla \varphi_{R}}{\left|\left(D v_{n}\right)^{a}\right|} d x+\int_{\mathbb{R}^{N}} V(\epsilon x) \varphi_{R}\left|v_{\epsilon}\right| d x \leq \int_{\mathbb{R}^{N}} f\left(v_{\epsilon}\right) v_{\epsilon} \varphi_{R} d x .
$$

By doing $R \rightarrow+\infty$ in both sides of (3.15) we get that

$$
\int_{\mathbb{R}^{N}}\left|D v_{\epsilon}\right|+\int_{\mathbb{R}^{N}} V(\epsilon x)\left|v_{\epsilon}\right| d x \leq \int_{\mathbb{R}^{N}} f\left(v_{\epsilon}\right) v_{\epsilon} d x
$$

what proves the claim.

By the Claim and (3.13), it follows that there exists $t_{\epsilon} \in(0,1]$ such that $t_{\epsilon} v_{\epsilon} \in \mathcal{N}_{\epsilon}$.

Note also that

$$
c_{\epsilon}+o_{n}(1)=\Phi_{\epsilon}\left(v_{n}\right)+o_{n}(1)=\Phi_{\epsilon}\left(v_{n}\right)-\Phi_{\epsilon}^{\prime}\left(v_{n}\right) v_{n}=\int_{\mathbb{R}^{N}}\left(f\left(v_{n}\right) v_{n}-F\left(v_{n}\right)\right) d x .
$$

Then applying Fatou Lemma in the last inequality together with $\left(f_{4}\right)$, it follows that

$$
\begin{aligned}
c_{\epsilon} & \geq \int_{\mathbb{R}^{N}}\left(f\left(v_{\epsilon}\right) v_{\epsilon}-F\left(v_{\epsilon}\right)\right) d x \\
& \geq \int_{\mathbb{R}^{N}}\left(f\left(t_{\epsilon} v_{\epsilon}\right) t_{\epsilon} v_{\epsilon}-F\left(t_{\epsilon} v_{\epsilon}\right)\right) d x \\
& =\Phi_{\epsilon}\left(t_{\epsilon} v_{\epsilon}\right)-\Phi_{\epsilon}^{\prime}\left(t_{\epsilon} v_{\epsilon}\right) t_{\epsilon} v_{\epsilon} \\
& =\Phi_{\epsilon}\left(t_{\epsilon} v_{\epsilon}\right) \\
& \geq c_{\epsilon} .
\end{aligned}
$$

Hence, $t_{\epsilon}=1, \Phi_{\epsilon}\left(v_{\epsilon}\right)=c_{\epsilon}$, and by (3.17)

$$
f\left(v_{n}\right) v_{n}-F\left(v_{n}\right) \rightarrow f\left(v_{\epsilon}\right) v_{\epsilon}-F\left(v_{\epsilon}\right) \text { in } L^{1}\left(\mathbb{R}^{N}\right) .
$$


This limit together with $\left(f_{4}\right)$ yield

$$
\begin{gathered}
f\left(v_{n}\right) v_{n} \rightarrow f\left(v_{\epsilon}\right) v_{\epsilon} \quad \text { in } L^{1}\left(\mathbb{R}^{N}\right) \\
F\left(v_{n}\right) \rightarrow F\left(v_{\epsilon}\right) \text { in } L^{1}\left(\mathbb{R}^{N}\right)
\end{gathered}
$$

and

$$
\left\|v_{n}\right\|_{\epsilon} \rightarrow\left\|v_{\epsilon}\right\|_{\epsilon},
$$

from where it follows that

$$
\left|v_{n}\right|_{1} \rightarrow\left|v_{\epsilon}\right|_{1} \text { as } n \rightarrow \infty .
$$

Here, we have used the fact that $\left(f_{4}\right)$ ensures that

$$
0 \leq(1-1 / \theta) f\left(v_{n}\right) v_{n} \leq f\left(v_{n}\right) v_{n}-F\left(v_{n}\right)
$$

and

$$
0 \leq(\theta-1) F\left(v_{n}\right) \leq f\left(v_{n}\right) v_{n}-F\left(v_{n}\right) .
$$

Then, by (3.18), we can apply the Lebesgue Dominated Convergence Theorem to get (3.19) and (3.20). Recalling that $\left\|v_{\epsilon}\right\|_{\epsilon}=\int_{\mathbb{R}^{N}} f\left(v_{\epsilon}\right) v_{\epsilon}$, the limit (3.19) combines with (3.9) to give (3.21).

As a consequence, since $\left(y_{n}\right)$ is a bounded sequence and $R_{n} \rightarrow+\infty$, the $L^{1}\left(\mathbb{R}^{N}\right)$ convergence of $\left(v_{n}\right)$ leads to

$$
\int_{B_{R_{n}}^{c}\left(y_{n}\right)}\left|v_{n}\right| d x \rightarrow 0 \text { as } n \rightarrow+\infty
$$

On the other hand, since $v_{n} \rightarrow v_{\epsilon} \neq 0$ in $L^{1}\left(\mathbb{R}^{N}\right)$ and by (3.11), it follows that

$$
\int_{B_{R_{n}}^{c}\left(y_{n}\right)}\left|v_{n}\right| d x \rightarrow(1-\alpha)\left|v_{\epsilon}\right|_{L^{1}\left(\mathbb{R}^{N}\right)}>0, \quad \text { as } n \rightarrow+\infty,
$$

a clear contradiction with (3.22).

- $\left(y_{n}\right)$ is unbounded:

In this case we should proceed as in the case where $\left(y_{n}\right)$ were bounded, but now dealing with the sequence $\left(\tilde{v}_{n}\right)$ where $\tilde{v}_{n}=v_{n}\left(\cdot-y_{n}\right)$. In fact, since $\left\|v_{n}\right\|=\left\|\tilde{v}_{n}\right\|$, it follows that $\left(\tilde{v}_{n}\right)$ is bounded and then converges, up to a subsequence, to some function $\tilde{v} \in B V\left(\mathbb{R}^{N}\right)$ in $L_{l o c}^{1}\left(\mathbb{R}^{N}\right)$, where $\tilde{v} \not \equiv 0$ by $(3.11)$.

\section{Claim 2}

$$
\Phi_{\infty}^{\prime}(\tilde{v}) \tilde{v} \leq 0 .
$$

In order to prove this claim, let us denote, for $v \in B V\left(\mathbb{R}^{N}\right)$,

$$
\|v\|_{\epsilon, y_{n}}:=\int_{\mathbb{R}^{N}}|D v|+\int_{\mathbb{R}^{N}} V\left(\epsilon x+\epsilon y_{n}\right)|v| d x
$$

and

$$
\Phi_{\epsilon, y_{n}}(v):=\|v\|_{\epsilon, y_{n}}-\int_{\mathbb{R}^{N}} F(v) d x .
$$

Note that, as before, $\Phi_{\epsilon, y_{n}}^{\prime}(v) w$ is well defined for all $v, w \in B V\left(\mathbb{R}^{N}\right)$ such that $(D w)^{s}$ is absolutely continuous w.r.t. $(D v)^{s}$ and $w$ is equal to 0 a.e. in the set where $v$ vanishes. Moreover, 


$$
\begin{aligned}
\Phi_{\epsilon, y_{n}}^{\prime}(v) w= & \int_{\mathbb{R}^{N}} \frac{(D v)^{a}(D w)^{a}}{\left|(D v)^{a}\right|} d x+\int_{\mathbb{R}^{N}} \frac{D v}{|D v|}(x) \frac{D w}{|D w|}(x)|(D w)|^{s} \\
& +\int_{\mathbb{R}^{N}} V\left(\epsilon x+\epsilon y_{n}\right) \operatorname{sgn}(v) w d x-\int_{\mathbb{R}^{N}} f(v) w d x
\end{aligned}
$$

Since $\int_{\mathbb{R}^{N}}\left|D v_{n}\right|=\int_{\mathbb{R}^{N}}\left|D \tilde{v}_{n}\right|$, from (3.3), by a change of variable, for all $w \in B V\left(\mathbb{R}^{N}\right)$ we have that

$$
\begin{aligned}
\left\|w\left(.+y_{n}\right)\right\|_{\epsilon, y_{n}}-\left\|\tilde{v}_{n}\right\|_{\epsilon, y_{n}} \geq & \int_{\mathbb{R}^{N}} f\left(\tilde{v}_{n}\right)\left(w\left(.+y_{n}\right)-\tilde{v}_{n}\right) d x \\
& -\tau_{n}\left(\int_{\mathbb{R}^{N}}\left|D\left(w\left(\cdot+y_{n}\right)-v_{n}\right)\right|+\int_{\mathbb{R}^{N}} V(\epsilon x\right. \\
& \left.\left.+\epsilon y_{n}\right)\left|w\left(.+y_{n}\right)-\tilde{v}_{n}\right| d x\right),
\end{aligned}
$$

For $\varphi_{R}:=\varphi(\cdot / R)$, where $\varphi \in C_{0}^{\infty}\left(\mathbb{R}^{N}\right), 0 \leq \varphi \leq 1, \varphi \equiv 1$ in $B_{R}(0)$ and $\varphi \equiv 0$ in $B_{2 R}(0)^{c}$, by taking in (3.25) $w(x)=v_{n}(x)+t \varphi_{R}\left(x-y_{n}\right) v_{n}(x)$ and making $t \rightarrow 0$, it follows that

$$
\Phi_{\epsilon, y_{n}}^{\prime}\left(\tilde{v}_{n}\right)\left(\varphi_{R} \tilde{v}_{n}\right)=o_{n}(1) .
$$

From (3.26), proceeding as in (3.15) and taking into account that $\left|y_{n}\right| \rightarrow+\infty$, we get that

$$
\int_{\mathbb{R}^{N}}|D \tilde{v}|+\int_{\mathbb{R}^{N}} V_{\infty}|\tilde{v}| d x \leq \int_{\mathbb{R}^{N}} f(\tilde{v}) \tilde{v} d x,
$$

which proves the claim.

By the Claim 2 and since $\tilde{v} \neq 0$, it follows that there exists $\tilde{t} \in(0,1]$ such that $\tilde{t} v \in \mathcal{N}_{\infty}$. On the other hand, note that

$$
\begin{aligned}
c_{\epsilon}+o_{n}(1) & =\Phi_{\epsilon}\left(v_{n}\right)+o_{n}(1) \\
& =\Phi_{\epsilon}\left(v_{n}\right)-\Phi_{\epsilon}^{\prime}\left(v_{n}\right) v_{n} \\
& =\int_{\mathbb{R}^{N}}\left(f\left(v_{n}\right) v_{n}-F\left(v_{n}\right)\right) d x \\
& =\int_{\mathbb{R}^{N}}\left(f\left(\tilde{v}_{n}\right) \tilde{v}_{n}-F\left(\tilde{v}_{n}\right)\right) d x .
\end{aligned}
$$

Then Fatou's Lemma gives

$$
\begin{aligned}
c_{\epsilon} & \geq \int_{\mathbb{R}^{N}}(f(\tilde{v}) \tilde{v}-F(\tilde{v})) d x \\
& \geq \int_{\mathbb{R}^{N}}(f(\tilde{t} \tilde{v}) \tilde{t} \tilde{v}-F(\tilde{t} \tilde{v})) d x \\
& =\Phi_{\infty}(\tilde{t} \tilde{v}) \\
& \geq c_{\infty},
\end{aligned}
$$

which contradicts Corollary 5.

Then we can conclude that Dichotomy in fact does not happen and then, it follows that Compactness must hold.

Claim $3\left(y_{n}\right)$ in (3.10) is a bounded sequence in $\mathbb{R}^{N}$.

Assuming this claim, for $\eta>0$, there exists $R>0$ such that, by (3.10),

$$
\int_{B_{R}^{c}(0)} \rho_{n} d x<\eta, \quad \forall n \in \mathbb{N},
$$


which is equivalent to

$$
\int_{B_{R}^{c}(0)}\left|v_{n}\right| d x \leq \eta\left|v_{n}\right|_{1} \leq C \eta, \quad \forall n \in \mathbb{N}
$$

Since $v_{\epsilon} \in L^{1}\left(\mathbb{R}^{N}\right)$, there exists $R_{0}>0$ such that

$$
\int_{B_{R_{0}}^{c}(0)}\left|v_{\epsilon}\right| d x \leq \eta
$$

Then, for $R_{1} \geq \max \left\{R, R_{0}\right\}$, since $v_{n} \rightarrow v_{\epsilon}$ in $L^{1}\left(B_{R_{1}}(0)\right)$, there exists $n_{0} \in \mathbb{N}$ such that

$$
\int_{B_{R_{1}}(0)}\left|v_{n}-v_{\epsilon}\right| d x \leq \eta \quad \forall n \geq n_{0} .
$$

Then, by (3.28), (3.29) and (3.30), it follows that if $n \geq n_{0}$,

$\int_{\mathbb{R}^{N}}\left|v_{n}-v_{\epsilon}\right| d x \leq \eta+\int_{B_{R_{1}}^{c}(0)}\left|v_{n}-v_{\epsilon}\right| d x \leq \eta+\int_{B_{R_{1}}^{c}(0)}\left|v_{n}\right| d x+\int_{B_{R_{1}}^{c}(0)}\left|v_{\epsilon}\right| d x \leq C_{1} \eta$.

Hence $v_{n} \rightarrow v_{\epsilon}$ in $L^{1}\left(\mathbb{R}^{N}\right)$ and since $\left(v_{n}\right)$ is bounded in $L^{1^{*}}\left(\mathbb{R}^{N}\right)$, by interpolation inequality it follows that

$$
v_{n} \rightarrow v_{\epsilon} \text { in } L^{q}\left(\mathbb{R}^{N}\right), \quad \text { for all } 1 \leq q<1^{*} .
$$

Now, what is left is proving Claim 3. However, the proof of it consist in suppose by contradiction that, up to a subsequence, $\left|y_{n}\right| \rightarrow+\infty$ and then proceed as in the case of Dichotomy, where $\left(y_{n}\right)$ were unbounded, reaching that $c_{\epsilon} \geq c_{\infty}$. But the latter is a clear contradiction when $\epsilon<\epsilon_{0}$, in the light of Corollary 5 .

Now let us just remark that if $\epsilon<\epsilon_{0}$, then $v_{\epsilon}$ is in fact a nontrivial solution of (2.1). First of all note that $(3.7),\left(f_{2}\right)$ and $\left(f_{3}\right)$ implies that

$$
\int_{\mathbb{R}^{N}} f\left(v_{n}\right) v_{n} d x \rightarrow \int_{\mathbb{R}^{N}} f\left(v_{\epsilon}\right) v_{\epsilon} d x, \text { as } n \rightarrow+\infty .
$$

Then from (3.3), (3.31) and the lower semicontinuity of $\|\cdot\|_{\epsilon}$ w.r.t. the $L^{1}\left(\mathbb{R}^{N}\right)$ convergence imply that

$$
\|w\|_{\epsilon}-\left\|v_{\epsilon}\right\|_{\epsilon} \geq \int_{\mathbb{R}^{N}} f\left(v_{\epsilon}\right)\left(w-v_{\epsilon}\right) d x, \quad \forall w \in B V\left(\mathbb{R}^{N}\right),
$$

and then $v_{\epsilon}$ is in fact a nontrivial solution of (2.1). Moreover, note that from (3.2)

$$
\begin{aligned}
c_{\epsilon} & \leq \Phi_{\epsilon}\left(v_{\epsilon}\right) \\
& =\Phi_{\epsilon}\left(v_{\epsilon}\right)-\Phi_{\epsilon}^{\prime}\left(v_{\epsilon}\right) v_{\epsilon} \\
& =\int_{\mathbb{R}^{N}}\left(f\left(v_{\epsilon}\right) v_{\epsilon}-F\left(v_{\epsilon}\right)\right) d x \\
& \leq \liminf _{n \rightarrow \infty} \int_{\mathbb{R}^{N}}\left(f\left(v_{n}\right) v_{n}-F\left(v_{n}\right)\right) d x \\
& =\Phi_{\epsilon}\left(v_{n}\right)+o_{n}(1) \\
& =c_{\epsilon} .
\end{aligned}
$$

Then $v_{\epsilon}$ is a ground-state solution of (2.1) and consequently $u_{\epsilon}=v_{\epsilon}(\cdot / \epsilon)$ is a ground-state bounded variation solution of (1.1). 


\subsection{Concentration behavior}

In the last section we have proved that for each $\epsilon \in\left(0, \epsilon_{0}\right)$, there exists $v_{\epsilon} \in B V\left(\mathbb{R}^{N}\right)$ solution of (2.1) such that $\Phi_{\epsilon}\left(v_{\epsilon}\right)=c_{\epsilon}$. Now let us show that this sequence of solutions concentrate around a global minimum of $V$. Before it, let us state and prove some preliminaries lemmas.

Lemma 8 There exist $\left\{y_{\epsilon}\right\}_{\epsilon>0} \subset \mathbb{R}^{N}$ and $R, \delta>0$ such that

$$
\liminf _{\epsilon \rightarrow 0} \int_{B_{R}\left(y_{\epsilon}\right)}\left|v_{\epsilon}\right| d x \geq \delta>0 .
$$

Proof In fact, on the contrary, thanks to [13, Theorem 1], it follows that $v_{\epsilon} \rightarrow 0$ in $L^{q}\left(\mathbb{R}^{N}\right)$ for all $1 \leq q<1^{*}$, as $\epsilon \rightarrow 0$. Then, by $\left(f_{2}\right),\left(f_{3}\right)$ and the Lebesgue Convergence Theorem, it follows that

$$
\int_{\mathbb{R}^{N}} f\left(v_{\epsilon}\right) v_{\epsilon} d x=o_{\epsilon}(1) .
$$

Taking $w=v_{\epsilon} \pm t v_{\epsilon}$ in (2.5) and passing to the limit as $t \rightarrow 0^{+}$, it follows that

$$
\left\|v_{\epsilon}\right\|_{\epsilon}=\int_{\mathbb{R}^{N}} f\left(v_{\epsilon}\right) v_{\epsilon} d x=o_{\epsilon}(1),
$$

which implies that $c_{\epsilon}=\Phi_{\epsilon}\left(v_{\epsilon}\right)=o_{\epsilon}(1)$, leading to a contradiction with Lemma 4 .

Lemma 9 The set $\left\{\epsilon y_{\epsilon}\right\}_{\epsilon>0}$ is bounded in $\mathbb{R}^{N}$.

Proof Suppose by contradiction that there exist $\epsilon_{n} \rightarrow 0$, such that $\left|\epsilon_{n} y_{n}\right| \rightarrow \infty$, as $n \rightarrow \infty$, where $y_{n}:=y_{\epsilon_{n}}$. In the following we proceed as in the proof of Claim 2 of Proposition 7. Let $v_{n}:=v_{\epsilon_{n}}$, and note that, if $\varphi_{R}$ is like in the proof of such claim, it follows that

$$
\Phi_{\epsilon_{n}, y_{n}}^{\prime}\left(\tilde{v}_{n}\right)\left(\varphi_{R} \tilde{v}_{n}\right)=0,
$$

where $\tilde{v}_{n}:=v_{n}\left(\cdot-y_{n}\right)$. As $\left(v_{n}\right),\left(\tilde{v}_{n}\right)$ is bounded in $B V\left(\mathbb{R}^{N}\right)$ and then $\tilde{v}_{n} \rightarrow \tilde{v}$ in $L_{l o c}^{1}\left(\mathbb{R}^{N}\right)$, up to a subsequence, where $\tilde{v} \neq 0$ by Lemma 8 . Then, as before, we get that

$$
\Phi_{\infty}^{\prime}(\tilde{v}) \tilde{v} \leq 0
$$

and then there exists $\tilde{t} \in(0,1]$ such that $\tilde{t} \tilde{v} \in \mathcal{N}_{\infty}$. Hence in the same way that in Claim 2 this will lead us to the contradiction that $c_{0}=\lim _{n \rightarrow \infty} c_{\epsilon_{n}} \geq c_{\infty}$.

Corollary 10 If $\epsilon_{n} \rightarrow 0$, then up to a subsequence, $\epsilon_{n} y_{n} \rightarrow y^{*}$ where

$$
V\left(y^{*}\right)=V_{0}=\inf _{\mathbb{R}^{N}} V .
$$

Proof If $\epsilon_{n} \rightarrow 0$, since by Lemma $9\left(\epsilon_{n} y_{n}\right)_{n \in \mathbb{N}}$ is bounded, then $\epsilon_{n} y_{n} \rightarrow y^{*} \in \mathbb{R}^{N}$ up to a subsequence. As in the proof of Claim 2 of Proposition 7 and of Lemma 9, it is possible to prove that

$$
c_{0}=\lim _{n \rightarrow \infty} c_{\epsilon_{n}} \geq c_{V\left(y^{*}\right)} \geq c_{0},
$$

where $c_{V\left(y^{*}\right)}$ is the mountain pass minimax level of problem (2.1) with $V\left(y^{*}\right)$ playing the role of $V(\epsilon x)$. Then it follows that $V\left(y^{*}\right)=\inf _{\mathbb{R}^{N}} V$. 
Lemma 11 If $\epsilon_{n} \rightarrow 0$, then there exists $\tilde{v} \in B V\left(\mathbb{R}^{N}\right)$ such that

$$
\tilde{v}_{n}:=v_{n}\left(\cdot-y_{n}\right) \rightarrow \tilde{v} \text { in } L_{l o c}^{1}\left(\mathbb{R}^{N}\right)
$$

and

$$
f\left(\tilde{v}_{n}\right) \tilde{v}_{n} \rightarrow f(\tilde{v}) \tilde{v} \text { in } L^{1}\left(\mathbb{R}^{N}\right) .
$$

Proof First of all, note that as in Lemma 6, it is possible to prove that $\left(\tilde{v}_{n}\right)$ is a bounded sequence in $B V\left(\mathbb{R}^{N}\right)$ and then that $\tilde{v}_{n} \rightarrow \tilde{v}$ in $L_{l o c}^{q}\left(\mathbb{R}^{N}\right)$ for all $1 \leq q<1^{*}$, where $\tilde{v} \in B V\left(\mathbb{R}^{N}\right)$. As in the proof of Lemma 9 , it is possible to prove that $\tilde{t} \in(0,1]$ such that $\tilde{t} \tilde{v} \in \mathcal{N}_{V\left(y^{*}\right)}=\mathcal{N}_{V_{0}}$ should verify $\tilde{t}=1$. Hence $\tilde{v} \in \mathcal{N}_{0}$ and note that $\Phi_{0}(\tilde{v})=c_{0}$. In fact

$$
\begin{aligned}
c_{0} & \leq \Phi_{0}(\tilde{v}) \\
& =\Phi_{0}(\tilde{v})-\Phi_{0}^{\prime}(\tilde{v}) \tilde{v} \\
& =\int_{\mathbb{R}^{N}}(f(\tilde{v}) \tilde{v}-F(\tilde{v})) d x \\
& \leq \liminf _{n \rightarrow \infty} \int_{\mathbb{R}^{N}}\left(f\left(\tilde{v}_{n}\right) \tilde{v}_{n}-F\left(\tilde{v}_{n}\right)\right) d x \\
& =\lim _{n \rightarrow \infty}\left(\Phi_{\epsilon_{n}}\left(v_{n}\right)-\Phi_{\epsilon_{n}}^{\prime}\left(v_{n}\right) v_{n}\right) \\
& =\lim _{n \rightarrow \infty} c_{\epsilon_{n}} \\
& =c_{0} .
\end{aligned}
$$

Then

$$
\lim _{n \rightarrow \infty} \int_{\mathbb{R}^{N}}\left(f\left(\tilde{v}_{n}\right) \tilde{v_{n}}-F\left(\tilde{v}_{n}\right)\right) d x=\int_{\mathbb{R}^{N}}(f(\tilde{v}) \tilde{v}-F(\tilde{v})) d x
$$

and hence $f\left(\tilde{v}_{n}\right) \tilde{v}_{n}-F\left(\tilde{v}_{n}\right) \rightarrow f(\tilde{v}) \tilde{v}-F(\tilde{v})$ in $L^{1}\left(\mathbb{R}^{N}\right)$. Thereby, by $\left(f_{4}\right)$,

$$
f\left(v_{n}\right) v_{n} \rightarrow f(\tilde{v}) \tilde{v} \quad \text { in } L^{1}\left(\mathbb{R}^{N}\right) .
$$

As a consequence of the last result, we can finish the proof of Theorem 1, by proving (1.2). In fact, if $\epsilon_{n} \rightarrow 0$, as $n \rightarrow \infty$, denoting $L=\int_{\mathbb{R}^{N}} f(\tilde{v}) \tilde{v} d x>0$, for a given $\delta>0$, by (3.34), there exists $R>0$ and $n_{0} \in \mathbb{N}$ such that, for $n \geq n_{0}$,

$$
\int_{B_{R}^{c}(0)} f\left(\tilde{v}_{n}\right) \tilde{v}_{n} d x<\delta .
$$

From which it follows that

$$
\int_{B_{R}(0)} f\left(\tilde{v}_{n}\right) \tilde{v}_{n} d x \geq L-\delta+o_{n}(1) .
$$

By the change of variable $\tilde{v}_{n}(x)=u_{n}\left(\epsilon_{n} x+\epsilon_{n} y_{n}\right),(3.35)$ and (3.36) imply that

$$
\int_{B_{\epsilon_{n} R}^{c}\left(\epsilon_{n} y_{n}\right)} f\left(u_{n}\right) u_{n} d x<\epsilon_{n}^{N} \delta
$$

and

$$
\int_{B_{\epsilon_{n} R}\left(\epsilon_{n} y_{n}\right)} f\left(u_{n}\right) u_{n} d x \geq C \epsilon_{n}^{N},
$$


for $n \geq n_{0}$, where $C>0$. Taking into account the fact that $\epsilon_{n} y_{n} \rightarrow x_{0}$ where $V\left(x_{0}\right)=V_{0}$, we can consider $\bar{R}>0$ such that, for $n \geq n_{0}, B_{R}\left(\epsilon_{n} y_{n}\right) \subset B_{\bar{R}}\left(x_{0}\right)$. Then from (3.37) and (3.38),

$$
\int_{B_{\epsilon_{n} \bar{R}}^{c}\left(x_{0}\right)} f\left(u_{n}\right) u_{n} d x<\epsilon_{n}^{N} \delta
$$

and

$$
\int_{B_{\epsilon_{n} \bar{R}}\left(x_{0}\right)} f\left(u_{n}\right) u_{n} d x \geq C \epsilon_{n}^{N},
$$

for $n \geq n_{0}$, what finishes de proof of Theorem 1 .

\section{Existence of solutions in the asymptotic constant case}

In this section we prove Theorem 2 and then we consider the assumptions $\left(f_{1}\right)-\left(f_{5}\right)$ and $\left(V_{1}\right)$ and $\left(V_{3}\right)$. As can be seen in the statement of Theorem 2, our existence result is independent of $\epsilon>0$ and then we can suppose without lack of generality that $\epsilon=1$. Then, in this section $\|\cdot\|_{1}$ denotes $\|\cdot\|_{\epsilon}$ when $\epsilon=1$.

Let us define $\Phi: B V\left(\mathbb{R}^{N}\right) \rightarrow \mathbb{R}$ by

$$
\Phi(u)=\int_{\mathbb{R}^{N}}|D u|+\int_{\mathbb{R}^{N}} V(x)|u| d x-\int_{\mathbb{R}^{N}} F(u) d x
$$

and consider $\Phi_{\infty}$ like in Sect. 2.

By the conditions on $f$, as in the last section, it is easy to see that $\Phi$ and $\Phi_{\infty}$ satisfy the geometric conditions of the Mountain Pass Theorem and then it is well defined the minimax levels

$$
\begin{aligned}
c & =\inf _{\gamma \in \Gamma} \sup _{t \in[0,1]} \Phi(\gamma(t)), \\
c_{\infty} & =\inf _{\gamma \in \Gamma \infty} \sup _{t \in[0,1]} \Phi_{\infty}(\gamma(t))
\end{aligned}
$$

where

$$
\Gamma=\left\{\gamma \in C\left([0,1], B V\left(\mathbb{R}^{N}\right)\right) ; \gamma(0)=0 \text { and } \Phi(\gamma(1))<0\right\}
$$

and

$$
\Gamma_{\infty}=\left\{\gamma \in C\left([0,1], B V\left(\mathbb{R}^{N}\right) ; \gamma(0)=0 \text { and } \Phi_{\infty}(\gamma(1))<0\right\} .\right.
$$

By $\left(V_{3}\right)$, it is easy to see that $\Phi(u) \leq \Phi_{\infty}(u)$ for all $u \in B V\left(\mathbb{R}^{N}\right)$ and as a consequence,

$$
c \leq c_{\infty} \text {. }
$$

By the results in $[12,13]$ together with the arguments explored in Sect. 5, it follows that there exists a critical point of $\Phi_{\infty}, w_{\infty} \in B V\left(\mathbb{R}^{N}\right)$, such that $\Phi_{\infty}\left(w_{\infty}\right)=c_{\infty}$. Also by [13], it is possible to define the Nehari manifolds associated to $\Phi$ and $\Phi_{\infty}$, respectively by

$$
\mathcal{N}=\left\{v \in B V\left(\mathbb{R}^{N}\right) \backslash\{0\} ; \Phi^{\prime}(v) v=0\right\}
$$

and

$$
\mathcal{N}_{\infty}=\left\{v \in B V\left(\mathbb{R}^{N}\right) \backslash\{0\} ; \Phi_{\infty}^{\prime}(v) v=0\right\}
$$


By the discussion in [12] it follows that $c=\inf _{\mathcal{N}} \Phi$ and $c_{\infty}=\inf _{\mathcal{N}_{\infty}} \Phi_{\infty}$. Moreover, it has been proved there that if there exists $u_{0} \in B V\left(\mathbb{R}^{N}\right)$ such that $\Phi\left(u_{0}\right)=\inf _{\mathcal{N}} \Phi$, then $u_{0}$ is a bounded variation solution of (1.1).

In order to effectively start with the proof of Theorem 2 let us consider the two possible cases about $c$ and $c_{\infty}$.

- Case 1: $c=c_{\infty}$. If this situation occurs, problem (1.1) has a ground state solution. In fact, since $w_{\infty} \in \mathcal{N}_{\infty}$, then

$$
\begin{aligned}
\int_{\mathbb{R}^{N}}\left|D w_{\infty}\right|+\int_{\mathbb{R}^{N}} V(x)\left|w_{\infty}\right| d x & \leq \int_{\mathbb{R}^{N}}\left|D w_{\infty}\right|+\int_{\mathbb{R}^{N}} V_{\infty}\left|w_{\infty}\right| d x \\
& =\int_{\mathbb{R}^{N}} f\left(w_{\infty}\right) w_{\infty} d x
\end{aligned}
$$

i.e.

$$
\Phi^{\prime}\left(w_{\infty}\right) w_{\infty} \leq 0
$$

Then there exists $t \in(0,1]$ such that $t w_{\infty} \in \mathcal{N}$. Hence, by $\left(f_{5}\right)$,

$$
\begin{aligned}
c & \leq \Phi\left(t w_{\infty}\right) \\
& =\Phi\left(t w_{\infty}\right)-\Phi^{\prime}\left(t w_{\infty}\right) t w_{\infty} \\
& =\int_{\mathbb{R}^{N}}\left(f\left(t w_{\infty}\right) t w_{\infty}-F\left(t w_{\infty}\right)\right) d x \\
& \leq \int_{\mathbb{R}^{N}}\left(f\left(w_{\infty}\right) w_{\infty}-F\left(w_{\infty}\right)\right) d x \\
& =\Phi_{\infty}\left(w_{\infty}\right) \\
& =c_{\infty} \\
& =c .
\end{aligned}
$$

This means that $t=1$ and $w_{\infty}$ is also a minimizer of $\Phi$ on $\mathcal{N}$ and then is a ground-state bounded variation solution of (1.1).

- Case 2: $c<c_{\infty}$. By [13, Theorem 4], there exists $\left(u_{n}\right) \subset B V\left(\mathbb{R}^{N}\right)$ such that

$$
\lim _{n \rightarrow \infty} \Phi\left(u_{n}\right)=c
$$

and

$$
\|w\|_{1}-\left\|u_{n}\right\|_{1} \geq \int_{\mathbb{R}^{N}} f\left(u_{n}\right)(w-u) d x-\tau_{n}\left\|w-u_{n}\right\|_{1}, \quad \forall w \in B V\left(\mathbb{R}^{N}\right),
$$

where $\tau_{n} \rightarrow 0$, as $n \rightarrow \infty$.

As in Lemma 6, it is possible to prove that $\left(u_{n}\right)$ is a bounded sequence in $B V\left(\mathbb{R}^{N}\right)$. By the compactness of the embeddings of $B V\left(\mathbb{R}^{N}\right)$ in $L_{l o c}^{q}\left(\mathbb{R}^{N}\right)$ for $1 \leq q<1^{*}$, it follows that there exists $u_{0} \in B V_{l o c}\left(\mathbb{R}^{N}\right)$ such that

$$
u_{n} \rightarrow u_{0} \text { in } L_{l o c}^{q}\left(\mathbb{R}^{N}\right) \quad \text { for } 1 \leq q<1^{*}
$$

and

$$
u_{n} \rightarrow u_{0} \text { a.e. in } \mathbb{R}^{N},
$$

as $n \rightarrow+\infty$. Note that as in the last section, it is possible to prove that $u_{0} \in B V\left(\mathbb{R}^{N}\right)$. Moreover, as in (3.8),

$$
\left|u_{n}\right|_{1} \nrightarrow 0, \quad \text { as } n \rightarrow+\infty \text {. }
$$


As in the proof of Proposition 7, let us use the Concentration of Compactness Principle due to Lions [20] to the following bounded sequence in $L^{1}\left(\mathbb{R}^{N}\right)$,

$$
\rho_{n}(x):=\frac{\left|u_{n}(x)\right|}{\left|u_{n}\right|_{1}} .
$$

By such a principle, one and only one of the following statements hold:

(Vanishing)

$$
\lim _{n \rightarrow+\infty} \sup _{y \in \mathbb{R}^{N}} \int_{B_{R}(y)} \rho_{n} d x=0, \forall R>0 ;
$$

(Compactness) There exist $\left(y_{n}\right) \subset \mathbb{R}^{N}$ such that for all $\eta>0$, there exists $R>0$ such that

$$
\int_{B_{R}\left(y_{n}\right)} \rho_{n} d x \geq 1-\eta, \quad \forall n \in \mathbb{N} ;
$$

(Dichotomy) There exist $\left(y_{n}\right) \subset \mathbb{R}^{N}, \alpha \in(0,1), R_{1}>0, R_{n} \rightarrow+\infty$ such that the functions $\rho_{n, 1}(x):=\chi_{B_{R_{1}}\left(y_{n}\right)}(x) \rho_{n}(x)$ and $\rho_{n, 2}(x):=\chi_{B_{R_{n}}^{c}\left(y_{n}\right)}(x) \rho_{n}(x)$ satisfy

$$
\int_{\mathbb{R}^{N}} \rho_{1, n} d x \rightarrow \alpha \text { and } \int_{\mathbb{R}^{N}} \rho_{2, n} d x \rightarrow 1-\alpha .
$$

Note that Vanishing does not occur, otherwise, by [13], it would hold that $\rho_{n} \rightarrow 0$ in $L^{q}\left(\mathbb{R}^{N}\right)$, for all $1 \leq q<1^{*}$. Taking (4.4) into account, this would imply that $u_{n} \rightarrow 0$ in $L^{q}\left(\mathbb{R}^{N}\right)$, for all $1 \leq q<1^{*}$ and then this would led us to $c=0$, a clear contradiction.

The case in which Dichotomy takes place, we get a contradiction in both situations, when $\left(y_{n}\right)$ is a bounded or an unbounded sequence, just repeating the arguments in the proof of Proposition 7.

Then it follows that Compactness holds and then, as in the proof of Claim 3 we can prove that $\left(y_{n}\right)$ is a bounded sequence. Then, for $\eta>0$, let $R>0$ such that (4.5) holds and note that this implies that

$$
\int_{B_{R}^{c}(0)} \rho_{n} d x<\eta, \quad \forall n \in \mathbb{N},
$$

which is equivalent to

$$
\int_{B_{R}^{c}(0)}\left|u_{n}\right| d x \leq \eta\left|u_{n}\right|_{1} \leq C \eta, \quad \forall n \in \mathbb{N} .
$$

Since $u_{0} \in L^{1}\left(\mathbb{R}^{N}\right)$, there exists $R_{0}>0$ such that

$$
\int_{B_{R_{0}}^{c}(0)}\left|u_{0}\right| d x \leq \eta .
$$

Then, for $R_{1} \geq \max \left\{R, R_{0}\right\}$, since $u_{n} \rightarrow u_{0}$ in $L^{1}\left(B_{R_{1}}(0)\right)$, there exists $n_{0} \in \mathbb{N}$ such that

$$
\int_{B_{R_{1}}(0)}\left|u_{n}-u_{0}\right| d x \leq \eta .
$$

Then, by (4.7), (4.8) and (4.9), it follows that if $n \geq n_{0}$,

$$
\int_{\mathbb{R}^{N}}\left|u_{n}-u_{0}\right| d x \leq \eta+\int_{B_{R_{1}}^{c}(0)}\left|u_{n}-u_{0}\right| d x \leq \eta+\int_{B_{R_{1}}^{c}(0)}\left|u_{n}\right| d x+\int_{B_{R_{1}}^{c}(0)}\left|u_{0}\right| d x \leq C_{1} \eta .
$$


Hence $u_{n} \rightarrow u_{0}$ in $L^{1}\left(\mathbb{R}^{N}\right)$ and since $\left(u_{n}\right)$ is bounded in $L^{1^{*}}\left(\mathbb{R}^{N}\right)$, by interpolation inequality it follows that

$$
u_{n} \rightarrow u_{0} \text { in } L^{q}\left(\mathbb{R}^{N}\right), \text { for all } 1 \leq q<1^{*} .
$$

From (4.10), $\left(f_{1}\right)$ and $\left(f_{2}\right)$ it follows that

$$
\int_{\mathbb{R}^{N}} f\left(u_{n}\right) u_{n} d x \rightarrow \int_{\mathbb{R}^{N}} f\left(u_{0}\right) u_{0} d x, \quad \text { as } n \rightarrow+\infty .
$$

Then from (3.3), (3.34) and the lower semicontinuity of $\|\cdot\|_{1}$ w.r.t. the $L^{1}\left(\mathbb{R}^{N}\right)$ convergence imply that

$$
\|w\|_{1}-\left\|u_{0}\right\|_{1} \geq \int_{\mathbb{R}^{N}} f\left(u_{0}\right)\left(w-u_{0}\right) d x, \quad \forall w \in B V\left(\mathbb{R}^{N}\right),
$$

and then $u_{0}$ is in fact a nontrivial solution of (1.1). Moreover, note that from (4.2)

$$
\begin{aligned}
c & \leq \Phi\left(u_{0}\right) \\
& =\Phi\left(v u_{0}\right)-\Phi^{\prime}\left(u_{0}\right) u_{0} \\
& =\int_{\mathbb{R}^{N}}\left(f\left(u_{0}\right) u_{0}-F\left(u_{0}\right)\right) d x \\
& \leq \liminf _{n \rightarrow \infty} \int_{\mathbb{R}^{N}}\left(f\left(u_{n}\right) u_{n}-F\left(u_{n}\right)\right) d x \\
& =\Phi\left(u_{n}\right)+o_{n}(1) \\
& =c .
\end{aligned}
$$

Then $u_{0}$ is a ground-state bounded variation solution of (1.1) and this finish the proof of Theorem 2.

\section{Existence of ground state solution for autonomous case}

In this short section, let us prove that there exists a ground-state solution to the autonomous problem

$$
\left\{\begin{array}{l}
-\Delta_{1} v+V_{\infty} \frac{v}{|v|}=f(v) \text { in } \mathbb{R}^{N}, \\
u \in B V\left(\mathbb{R}^{N}\right) .
\end{array}\right.
$$

Let $\Phi_{\infty}, c_{\infty}$ and $\mathcal{N}_{\infty}$ defined as in Sect. 3. By [13, Theorem 1.4], there exists $\left(w_{n}\right) \subset$ $B V\left(\mathbb{R}^{N}\right)$ such that $\Phi_{\infty}\left(w_{n}\right) \rightarrow c_{\infty}$ and moreover,

$$
\left\|w_{n}\right\|_{\infty}-\left\|w_{n}\right\|_{\infty} \geq \int_{\mathbb{R}^{N}} f\left(w_{n}\right)\left(w-w_{n}\right) d x-\tau_{n}\left\|w-v_{n}\right\|_{\epsilon}, \quad \forall w \in B V\left(\mathbb{R}^{N}\right) .
$$

where $\tau_{n} \rightarrow 0$, as $n \rightarrow \infty$.

As in the proof of Lemma 6 , it is possible to prove that $\left(w_{n}\right)$ is a bounded sequence in $B V\left(\mathbb{R}^{N}\right)$ and then $w_{n} \rightarrow w_{\infty}$ in $L_{l o c}^{q}\left(\mathbb{R}^{N}\right)$, where $1 \leq q<1^{*}$. It follows as in (3.6) that $w_{\infty} \in B V\left(\mathbb{R}^{N}\right)$. Also, there exist $R, \beta>0$ and a sequence $\left(y_{n}\right) \subset \mathbb{R}^{N}$ such that

$$
\liminf _{n \rightarrow+\infty} \int_{B_{R}\left(y_{n}\right)}\left|w_{n}\right| d x \geq \beta \text {. }
$$

In fact, otherwise, by [13, Theorem 1.1], $w_{n} \rightarrow 0$ in $L^{q}\left(\mathbb{R}^{N}\right)$ for $1<q<1^{*}$ and then, by $\left(f_{2}\right)$ and $\left(f_{3}\right), \Phi_{\infty}\left(w_{n}\right) \rightarrow 0$, leading to a clear contradiction with the fact that $c_{\infty}>0$. As $\Phi_{\infty}$ is invariant by translation, without loss of generality we can assume that $w_{\infty} \neq 0$. 
By proceeding exactly as in the proof of Proposition 7, Claim 1, it follows that $\Phi_{\infty}^{\prime}\left(w_{\infty}\right) w_{\infty} \leq 0$. Then there exists $t_{\infty} \in(0,1]$ such that $t_{\infty} w_{\infty} \in \mathcal{N}_{\infty}$. Note also that $c_{\infty}+o_{n}(1)=\Phi_{\infty}\left(w_{n}\right)+o_{n}(1)=\Phi_{\infty}\left(w_{n}\right)-\Phi_{\infty}^{\prime}\left(w_{n}\right) w_{n}=\int_{\mathbb{R}^{N}}\left(f\left(w_{n}\right) w_{n}-F\left(w_{n}\right)\right) d x$.

Then applying Fatou's Lemma in the last inequality together with $\left(f_{4}\right)$, it follows that

$$
\begin{aligned}
c_{\infty} & \geq \int_{\mathbb{R}^{N}}\left(f\left(w_{\infty}\right) w_{\infty}-F\left(w_{\infty}\right)\right) d x \\
& \geq \int_{\mathbb{R}^{N}}\left(f\left(t_{\infty} w_{\infty}\right) t_{\infty} w_{\infty}-F\left(t_{\infty} w_{\infty}\right)\right) d x \\
& =\Phi_{\infty}\left(t_{\infty} w_{\infty}\right)-\Phi_{\infty}^{\prime}\left(t_{\infty} w_{\infty}\right) t_{\infty} w_{\infty} \\
& =\Phi_{\infty}\left(t_{\infty} w_{\infty}\right) \\
& \geq c_{\infty}
\end{aligned}
$$

Hence, $t_{\infty}=1, \Phi_{\infty}\left(w_{\infty}\right)=c_{\infty}$ and, by [12, Theorem 5], it follows that $w_{\infty}$ is a ground-state bounded variation solution of (5.1).

Acknowledgements M.T.O. Pimenta has been supported by FAPESP 2017/01756-2 and CNPq/Brazil 442520/2014-0. C.O. Alves was partially supported by CNPq/Brazil 304036/2013-7 and INCT-MAT. The authors would like to thank to the referee for his/her important comments.

\section{References}

1. Ambrosetti, A., Badiale, M., Cingolani, S.: Semiclassical states of nonlinear Schrödinger equations. Arch. Ration. Mech. Anal. 140, 285-300 (1997)

2. Anzellotti, G.: The Euler equation for functionals with linear growth. Trans. Am. Math. Soc. 290(2), 483-501 (1985)

3. Attouch, H., Buttazzo, G., Michaille, G.: Variational Analysis in Sobolev and BV Spaces: Applications to PDEs and Optimization. MPS-SIAM, Philadelphia (2006)

4. Bartle, R.: The Elements of Integration and Lebesgue Measure. Wiley, New York (1995)

5. Chang, K.: Variational methods for non-differentiable functionals and their applications to partial differential equations. J. Math. Anal. Appl. 80, 102-129 (1981)

6. Chang, K.: The spectrum of the 1-Laplace operator. Commun. Contemp. Math. 9(4), 515-543 (2009)

7. Cingolani, S., Lazzo, M.: Multiple semiclassical standing waves for a class of nonlinear Schrödinger equations. Topol. Methods Nonlinear Anal. 10, 1-13 (1997)

8. del Pino, M., Felmer, P.L.: Local mountain pass for semilinear elliptic problems in unbounded domains. Calc. Var. Partial Differ. Equ. 4(2), 121-137 (1996)

9. Clarke, F.: Generalized gradients and applications. Trans. Am. Math. Soc. 205, 247-262 (1975)

10. Degiovanni, M., Magrone, P.: Linking solutions for quasilinear equations at critical growth involving the 1-Laplace operator. Calc. Var. Partial Differ. Equ. 36, 591-609 (2009)

11. Evans, L., Gariepy, R.: Measure Theory and Fine Properties of Functions. CRC Press, Boca Raton (1992)

12. Figueiredo, G.M., Pimenta, M.T.O.: Nehari method for locally Lipschitz functionals with examples in problems in the space of bounded variation functions. arXiv:1704.03748v1 [math.AP]

13. Figueiredo, G.M., Pimenta, M.T.O.: Strauss' and Lions' type results in $B V\left(\mathbb{R}^{N}\right)$ with an application to 1-Laplacian problem. arXiv:1610.07369v1

14. Figueiredo, G.M., Pimenta, M.T.O.: Existence of bounded variation solutions for a 1-Laplacian problem with vanishing potentials. arXiv:1609.06683

15. Floer, A., Weinstein, A.: Nonspreading wave packets for the cubic Schrödinger equation with a bounded potential. J. Funct. Anal. 69(3), 397-408 (1986)

16. Gui, C.: Existence of multi-bump solutions for nonlinear Schrödinger equations via variational method. Comm. Partial Differ. Equ. 21(5-6), 787-820 (1996)

17. Giusti, E.: Minimal Surfaces and Functions of Bounded Variation. Birkhäuser, Boston (1984) 
18. Jianfu, Y., Xiping, Z.: On the existence of nontrivial solution of quasilinear elliptic boundary value problem for unbounded domains. Acta Math. Sci. 7(3), 341-359 (1987)

19. Kawohl, B., Schuricht, F.: Dirichlet problems for the 1-Laplace operator, including the eigenvalue problem. Commun. Contemp. Math. 9(4), 525-543 (2007)

20. Lions, P.L.: The concentration-compactness principle in the calculus of variations. The Locally compact case, part 2. Analles Inst. H. Poincaré Sect. C 1, 223-283 (1984)

21. Oh, Y.G.: On positive multi-lump bound states of nonlinear Schrödinger equations under multiple well potential. Commun. Math. Phys. 131(2), 223-253 (1990)

22. Rabinowitz, P.H.: On a class of nonlinear Schrödinger equations. Z. Angew. Math. Phys. 43(2), 270-291 (1992)

23. Szulkin, A.: Minimax principle for lower semicontinuous functions and applications to nonlinear boundary value problems. Ann. Inst. H. Poincaré 3(2), 77-109 (1986)

24. Wang, X.: On concentration of positive bound states of nonlinear Schrödinger equations. Commun. Math. Phys. 153(2), 229-244 (1993) 